\title{
The weak coupling limit for the random Schrödinger equation: The average wave function
}

\author{
Thomas Chen* Tomasz Komorowski ${ }^{\dagger} \quad$ Lenya Ryzhik ${ }^{\ddagger}$
}

September 25, 2018

\begin{abstract}
We consider the Schrödinger equation with a time-independent weakly random potential of a strength $\varepsilon \ll 1$, with Gaussian statistics. We prove that when the initial condition varies on a scale much larger than the correlation length of the potential, the compensated wave function converges to a deterministic limit on the time scale $t \sim \varepsilon^{-2}$. This is shown under the sharp assumption that the correlation function $R(x)$ of the random potential decays slower than $1 /|x|^{2}$, which ensures that the effective potential is finite. When $R(x)$ decays slower than $1 /|x|^{2}$ we establish an anomalous diffusive behavior for the averaged wave function on a time scale shorter than $\varepsilon^{-2}$, as long as the initial condition is "sufficiently macroscopic". We also consider the kinetic regime when the initial condition varies on the same scale as the random potential and obtain the limit of the averaged wave function for potentials with the correlation functions decaying faster than $1 /|x|^{2}$. We use random potentials of the Schonberg class which allows us to bypass the oscillatory phase estimates.
\end{abstract}

\section{Introduction}

We consider the large time behavior of the solutions of the weakly random Schrödinger equation

$$
i \frac{\partial \psi}{\partial t}+\frac{1}{2} \Delta \psi-\varepsilon V(x) \psi=0, \quad t>0, \quad x \in \mathbb{R}^{d} .
$$

Here, the random potential $V(x)$ is a mean-zero Gaussian statistically homogeneous random field over a probability space $(\Omega, \mathcal{V}, \mathbb{P})$ with the covariance function $R(x)$ :

$$
R(x)=\mathbb{E}(V(y) V(x+y)) .
$$

\footnotetext{
*Department of Mathematics, University of Texas at Austin, 2515 Speedway C1200, Austin, TX 78712, USA; tc@math.texas.edu

${ }^{\dagger}$ Institute of Mathematics, Maria Curie Sklodowska University, pl. Marii Curie Sklodowskiej 1, 20-031 Lublin, Poland; komorow@hektor.umcs.lublin.pl

${ }_{\ddagger}^{\ddagger}$ Department of Mathematics, Stanford University, Stanford, CA 94305, USA; ryzhik@stanford.edu
} 
We denote by $\mathbb{E}$ the expectation with respect to $\mathbb{P}$. The small parameter $\varepsilon \ll 1$ measures the relative strength of the random fluctuations. The initial condition for (1.1):

$$
\psi(0, x)=\psi_{0}\left(\frac{x}{l_{i}}\right)
$$

varies on a scale $l_{i}$. Scale-wise, it is implicitly assumed in (1.1) that the random potential $V(x)$ varies on a scale $l_{c}=O(1)$.

The Schrödinger equation (1.1) preserves the total mass

$$
M(t)=\int_{\mathbb{R}^{d}}|\psi(t, x)|^{2} d x=M(0)
$$

and the total energy

$$
E(t)=\int_{\mathbb{R}^{d}}\left[\frac{1}{2}|\nabla \psi(t, x)|^{2}+\varepsilon V(x)|\psi(t, x)|^{2}\right] d x=\int_{\mathbb{R}^{d}}|\xi|^{2}|\hat{\psi}(t, \xi)|^{2} \overline{d \xi}+\varepsilon \int_{\mathbb{R}^{d}} V(x)|\psi(t, x)|^{2} d x .
$$

Both here and in what follows we denote $d p:=d p /(2 \pi)^{d}$. We also use the notation

$$
\hat{\psi}(t, \xi)=\int_{\mathbb{R}^{d}} e^{-i \xi \cdot x} \psi(t, x) d x
$$

for the Fourier transform of the wave function.

Since the random potential is weak, the preservation of the energy, together with the mass conservation, means, approximately, that the bulk of the energy would remain at the frequency scale $|\xi| \sim l_{i}^{-1}$ of the initial condition.

\section{The kinetic regime}

This problem has been extensively studied in the past when the random potential is rapidly decorrelating and the initial condition varies on the same scale as the random potential. In other words, $l_{i}=1$, or, in the dimensional variables, $l_{i}=l_{c}$. This is sometimes known as the kinetic regime, and is particularly interesting since it leads to a full interaction between the random fluctuations and the wave function. The first key result here is by Spohn [23] who considered the rescaled wave function

$$
\psi_{\varepsilon}(t, x)=\psi\left(\frac{t}{\varepsilon^{2}}, \frac{x}{\varepsilon^{2}}\right)
$$

and its Wigner transform

$$
W_{\varepsilon}(t, x, \xi)=\int_{\mathbb{R}^{d}} e^{i \xi \cdot y} \psi_{\varepsilon}\left(t, x-\frac{\varepsilon^{2} y}{2}\right) \psi_{\varepsilon}^{*}\left(t, x+\frac{\varepsilon^{2} y}{2}\right) d y
$$

Here, $z^{*}$ denotes the complex conjugate of $z \in \mathbb{C}$. The main result of [23] is that, if the correlation function $R(x)$ is smooth and sufficiently rapidly decaying, then $\mathbb{E}\left(W_{\varepsilon}(t, x, \xi)\right)$ converges, 
as $\varepsilon \rightarrow 0$, in the sense of distributions to the solution $W(t, x, \xi)$ of the radiative transport equation

$$
\frac{\partial W(t, x, \xi)}{\partial t}+\xi \cdot \nabla_{\xi} W(t, x, \xi)=\int_{\mathbb{R}^{d}} \hat{R}(\xi-p) \delta\left(\frac{|\xi|^{2}-|p|^{2}}{2}\right)(W(t, x, p)-W(t, x, \xi)) \frac{d p}{(2 \pi)^{d-1}}
$$

Here, $\hat{R}(p)$ is the power energy spectrum, the Fourier transform of the correlation function $R(x)$. It follows that the average density of the solution has the weak limit

$$
\lim _{\varepsilon \rightarrow 0+} \mathbb{E}\left|\psi_{\varepsilon}(t, x)\right|^{2}=\int_{\mathbb{R}^{d}} W(t, x, \xi) d \xi
$$

This result has been established in [23] in dimensions $d \geq 3$, on a finite time interval $0 \leq t \leq T$ with $T$ that is independent of $\varepsilon$ (thus, in the original microscopic variables the time interval is $\left.T / \varepsilon^{2}\right)$ but that does depend on the correlation function $R(x)$. The assumption that the power energy spectrum satisfies $\hat{R} \in L^{1} \cap L^{\infty}$ is essential for the estimates in [23].

The kinetic limit has been further studied by L. Erdös and H. T. Yau in [8]. They have removed the restriction of a finite time interval convergence, and have shown that the kinetic limit holds on any finite time interval $0 \leq t \leq T$. That is, microscopically, it is valid on any time interval of the size $O\left(\varepsilon^{-2}\right)$. The assumptions on the decay of the correlation function in [7, 8] are more stringent than in [23]. See also [2, 3, 6, 19, 20] for related results. Convergence of the expectation has been strengthened to the $L^{2}$-convergence in [7], with some further improvements obtained in [4].

The results of [8] were subsequently extended to the analysis of the diffusive limit in [10, 11]. For random Schrödinger equations coupled to thermal noise, diffusive limits have been studied in [12, 13, 17].

\section{The homogenization regime}

Another regime recently investigated by G. Bal and N. Zhang in [24, 25] is $l_{i}=\varepsilon^{-1} l_{c}$. That is, the initial condition for (1.1) is of the form $\psi(0, x)=\psi_{0}(\varepsilon x)$. This is an interesting and convenient regime as in this case the central limit time scale $O\left(\varepsilon^{-2}\right)$, which comes from the size $\varepsilon$ of the random potential, matches the homogeneous Schrödinger time scale $t \sim l_{i}^{2}$, which is the time for the linear "Schrödinger phase" $\exp \left(i t|\xi|^{2} / 2\right)$ to become of the order $O(1)$ for $|\xi| \sim l_{i}^{-1}$. Accordingly, when $l_{i}=\varepsilon^{-1}$, a natural object is the rescaled wave function

$$
\psi_{\varepsilon}(t, x)=\psi\left(\frac{t}{\varepsilon^{2}}, \frac{x}{\varepsilon}\right)
$$

It has been shown in [25] in $d \geq 3$ that $\psi_{\varepsilon}(t, x)$ converges in probability, as $\varepsilon \rightarrow 0$ to the solution of the deterministic homogenized problem

$$
i \frac{\partial \psi_{*}}{\partial t}+\frac{1}{2} \Delta \psi_{*}-R_{*} \psi_{*}=0
$$


with the initial condition $\psi_{*}(0, x)=\psi_{0}(x)$. The effective potential in (1.7) is simply a constant

$$
R_{*}=\int_{\mathbb{R}^{d}} \frac{\hat{R}(p)}{|p|^{2}} đ p .
$$

Thus, there is a substantial difference in the behavior of the solutions when $l_{i}=1$ and $l_{i}=\varepsilon^{-1}$. In both cases, the solution is affected in a non-trivial way by the central limit time scale $t \sim \varepsilon^{-2}$. However, in the former case, the solution behaves stochastically on this time scale - this is the kinetic regime, while in the latter it has a deterministic behavior - this is the homogenization regime.

A stochastic (non-deterministic) limit for such problems when the Laplacian is replaced by a higher power $(-\Delta)^{m}$ with $m>1$ has been investigated in [24], also for rapidly decorrelating potentials.

\section{The main results}

Our main interest in this paper is in the breakdown of the homogenization regime when the effective potential $R_{*}$ in (1.8) is infinite. However, all of the above results have been established under much more stringent assumptions on the correlation function than just

$$
R_{*}<+\infty
$$

Hence, we first carry out the analysis of the behavior of the wave function only assuming (1.9), to reach the threshold of the validity of the homogenization and kinetic regimes. We also obtain some results when $R_{*}=+\infty$, and these regimes break down.

\section{The correlation function}

In order to perform the analysis under weaker assumptions on the decay of the correlation function than previously, we take a special form of the correlation function. We shall assume that the power energy spectrum of the potential $V(x)$ is positive definite in the sense of Schoenberg [22]. That is, $\hat{R}(p)$ is of the form

$$
\hat{R}(p)=\int_{1}^{+\infty} e^{-\lambda^{2}|p|^{2} / 2} s(\lambda) \frac{d \lambda}{\lambda^{\gamma}},
$$

with a positive bounded function $s(\lambda)$ that satisfies:

$$
0<c \leq s(\lambda) \leq c^{-1}, \quad \forall \lambda>1
$$

for some $c>0$. It is easy to see that this assumption is not restrictive in term of the spatial decay of the correlation function. The power spectrum has the asymptotics

$$
\hat{R}(p) \sim|p|^{\gamma-1}, \quad|p| \ll 1,
$$


which translates into the following spatial decay for the correlation function itself:

$$
R(x) \sim \frac{1}{|x|^{d+\gamma-1}}, \quad|x| \gg 1 .
$$

Since the potential $V(x)$ is a Gaussian random field, we have $\mathbb{E}\left(V^{2}(x)\right)<+\infty$, thus $\hat{R} \in L^{1}\left(\mathbb{R}^{d}\right)$, and (1.11) implies that $\gamma>1-d$. On the other hand, choosing various $\gamma>1-d$ in (1.10), we may achieve an arbitrarily slow or fast decay of the correlation function in (1.12). A straightforward computation shows that the effective potential in (1.8) is given by

$$
R_{*}=\frac{1}{(2 \pi)^{d / 2}(d-2)} \int_{1}^{\infty} \frac{s(\lambda) d \lambda}{\lambda^{\gamma+d-2}} .
$$

Thus, the effective potential is finite: $R_{*}<+\infty$, provided that $\gamma>3-d$, or, in terms of the decay of the correlation function, that

$$
R(x) \sim \frac{1}{|x|^{m}}, \quad|x| \gg 1,
$$

with some $m>2$. This assumption does not depend on the spatial dimension.

We should mention that, similarly to the very technical proofs in [8, 23], our strategy relies on the Duhamel expansion of the solutions of the Schrödinger equation, and summation of the Feynman diagram expansions that come up after we evaluate various expectations. Typically, this requires intricate oscillatory phase estimates. One contribution of this paper is a simple observation that for the random potentials that are in the Schoenberg class, we may bypass the oscillatory phase arguments. Instead, one needs to estimate some quite explicit determinants, simplifying substantially the analysis and allowing it to go a bit deeper.

\section{The microscopic initial conditions}

As has been observed in [1], it is convenient to take out the rapidly growing phase $\exp \left(i t|\xi|^{2} t / 2\right)$ and consider the compensated wave function

$$
\hat{\zeta}(t, \xi):=\hat{\psi}(t, \xi) e^{i|\xi|^{2} t / 2} .
$$

As long as $R_{*}<+\infty$, we will be interested in the central limit time scales $t \sim \varepsilon^{-2}$, and, accordingly, define

$$
\hat{\zeta}_{\varepsilon}(t, \xi):=\hat{\psi}\left(\frac{t}{\varepsilon^{2}}, \xi\right) e^{i|\xi|^{2} t /\left(2 \varepsilon^{2}\right)} .
$$

We first consider the initial condition for (1.1) with $l_{i}=1$ - this is the kinetic regime considered in [8, 23], or, alternatively, the case of microscopic initial conditions. Let us define

$$
r(\xi)=\frac{i}{2(2 \pi)^{d / 2}} \int_{1}^{\infty} \frac{s(\lambda) \kappa(\xi, \lambda) d \lambda}{\lambda^{\gamma+d-2}},
$$


with

$$
\kappa(\xi, \lambda):=\int_{0}^{+\infty} \frac{1}{(1+i \tau)^{d / 2}} \exp \left\{-\frac{(\lambda|\xi| \tau)^{2}}{2(1+i \tau)}\right\} d \tau .
$$

It is straightforward to verify that the real part of $r(\xi)$ is the total scattering cross-section in the radiative transport equation (1.5)).

Theorem 1.1 Suppose that $d \geq 3$ and $\gamma>3-d$. Let $\psi(t, x)$ be the solution of (1.1) with the initial condition $\psi(0, x)=\psi_{0}(x) \in \mathcal{S}\left(\mathbb{R}^{d}\right)$. Then, there exists $t_{0}>0$ such that for all $t \in\left[0, t_{0}\right]$ and $\xi \in \mathbb{R}^{d}$, we have

$$
\lim _{\varepsilon \rightarrow 0+} \mathbb{E} \hat{\zeta}_{\varepsilon}(t, \xi)=\hat{\psi}_{0}(\xi) \exp \{i r(\xi) t\} .
$$

This result is not surprising - the imaginary part of $r(\xi)$ agrees with the total scattering crosssection for the kinetic equation obtained in [8, 23]. However, in terms of the assumptions on the correlation function $R(x)$, it holds up to the threshold $\gamma=3-d$, when the effective potential becomes infinite. On the other hand, as $r(0)=-R_{*}$, the result cannot be true (at least for $\xi=0)$ when $\gamma \leq 3-d$, since in that case $R_{*}=+\infty$. Let us add that the assumption that $\psi_{0}$ is in the Schwartz class can be easily improved but it is not the focus of this paper.

Preliminary computations indicate that the convergence of the expectation in Theorem 1.1 may be bootstrapped to the convergence of the second moment, recovering, in addition, the kinetic limit of [8, 23]. One may also combine the techniques of the present paper with the strategy of [8] to extend the result to all times $t_{0}>0$. However, to keep the paper relatively short, we postpone these directions for a future investigation.

\section{The macroscopic initial conditions: homogenization}

Unlike the microscopic initial conditions with the width $l_{i}=1$, the macroscopic initial conditions have the initial pulse width $l_{i}=\varepsilon^{-\beta} \gg l_{c}=1$, with some $\beta>0$. Recall that the special case $\beta=1$ has been considered in [25] for very rapidly decorrelating potentials. In other words, the initial condition for (1.1) is of the form

$$
\psi(0, x)=\varepsilon^{d \beta / 2} \psi_{0}\left(\varepsilon^{\beta} x\right),
$$

with $\psi_{0} \in \mathcal{S}\left(\mathbb{R}^{d}\right)$. The pre-factor in (1.20) is introduced simply to keep the $L^{2}$ norm of the solution to be of order $O(1)$. Its Fourier transform is

$$
\hat{\psi}(0, \xi)=\varepsilon^{-d \beta / 2} \hat{\psi}_{0}\left(\varepsilon^{-\beta} \xi\right) .
$$

To take into account the lower frequencies of the macroscopic initial conditions, and the aforementioned fact that the bulk of the energy is expected to stay at the original frequency, the compensated wave function $\hat{\zeta}_{\varepsilon}(t, \xi)$ on the time scales $t \sim \varepsilon^{-2}$ is now defined as

$$
\hat{\zeta}_{\varepsilon}(t, \xi):=\varepsilon^{d \beta / 2} \hat{\psi}\left(\frac{t}{\varepsilon^{2}}, \varepsilon^{\beta} \xi\right) e^{i \varepsilon^{2(\beta-1)}|\xi|^{2} t / 2} .
$$


This allows us to track frequencies of the order $O\left(\varepsilon^{\beta}\right)$, present in the initial condition, at the central limit time scale $t \sim O\left(\varepsilon^{-2}\right)$.

Theorem 1.2 Suppose that $d \geq 3$ and $\gamma>3-d$ and the initial condition for (1.1) is of the form (1.20). Then, there exists $t_{0}>0$ such that for all $t \in\left[0, t_{0}\right]$ we have

$$
\lim _{\varepsilon \rightarrow 0+} \mathbb{E}\left\|\hat{\zeta}_{\varepsilon}(t, \cdot)-\bar{\zeta}(t, \cdot)\right\|_{L^{2}\left(\mathbb{R}^{d}\right)}=0
$$

with

$$
\bar{\zeta}(t, \xi):=\hat{\psi}_{0}(\xi) \exp \left\{-i R_{*} t\right\}
$$

with the effective potential $R_{*}$ given by (1.13).

Thus, the homogenization result of [25] holds not just for the somewhat artificial choice $l_{i}=\varepsilon^{-1}$ but essentially for all $l_{i} \gg 1$. The threshold to the stochastic behavior is exactly at $l_{i}=l_{c}$. The result of Theorem 1.2 is sharp in terms of the assumptions on the correlation function its conclusion holds for all random potentials such that the effective potential $R_{*}<+\infty$.

\section{The macroscopic initial data: superdiffusive behavior}

Next, we consider slowly decorrelating random potentials, with the power spectrum of the form (1.10), and $\gamma<3-d$, so that $R(x)$ decays at a rate slower than $1 /|x|^{2}$ as $|x| \rightarrow+\infty-$ see (1.12). Then, the effective potential is infinite: $R_{*}=+\infty$, and the homogenization limit can not hold. We assume that the initial condition is macroscopic: $l_{i}=\varepsilon^{-\beta}$ with some $\beta>0$. Typically, in such situations one expects a non-trivial effect of the random fluctuations to be seen on a time scale $t \sim \varepsilon^{-2 \alpha}$ with some $\alpha \in(0,1)$, rather than for $t \sim \varepsilon^{-2}$. Accordingly, the compensated wave function $\hat{\zeta}_{\varepsilon}(t, \xi)$ is now defined as

$$
\hat{\zeta}_{\varepsilon}(t, \xi):=\varepsilon^{d \beta / 2} \hat{\psi}^{(\varepsilon)}\left(\frac{t}{\varepsilon^{2 \alpha}}, \varepsilon^{\beta} \xi\right) e^{i \varepsilon^{2(\beta-\alpha)}|\xi|^{2} t / 2}
$$

We denote the standard Brownian motion by $B_{t}$ and the expectation with respect to it by $\mathbb{M}$.

Theorem 1.3 Suppose that $d \geq 3$ and $\beta>\alpha$, where

$$
\alpha:=\frac{2}{5-\gamma-d}
$$

and $\gamma \in(1-d, 3-d)$. Then, there exists $t_{0}>0$ such that

$$
\bar{\zeta}(t, \xi):=\lim _{\varepsilon \rightarrow 0+} \mathbb{E} \hat{\zeta}_{\varepsilon}(t, \xi)=\hat{\psi}_{0}(\xi) \mathbb{M} \exp \left\{-\frac{\mathfrak{R} t^{H}}{4} \int_{0}^{1} \int_{0}^{1}\left|B_{s}-B_{s^{\prime}}\right|^{1-\gamma-d} d s d s^{\prime}\right\}
$$

for all $t \in\left[0, t_{0}\right]$ with $H=1 / \alpha$, and a constant $\mathfrak{R}$ whose real part $\operatorname{Re} \mathfrak{R}>0$. 
The assumption $\beta>\alpha$ informally means that the initial condition is "very macroscopic". A short computation, starting from (1.26) shows that there exists $C>0$ such that

$$
|\bar{\zeta}(t, \xi)| \leq C \exp \left\{-t^{H_{*}} / C\right\}, \quad(t, \xi) \in[0,+\infty) \times \mathbb{R}^{d}
$$

with

$$
H_{*}=\frac{5-\gamma-d}{\gamma+d+1}
$$

Note that the randomization time $t^{-2 \alpha}$ does not depend on $\beta$, as long as $\beta>\alpha$. Informally, this means that solutions with all sufficiently slowly varying initial conditions are randomized at the same time scale $t^{-2 \alpha}$. We expect that solutions with the "less macroscopic" initial conditions varying on a scale $l_{i}=\varepsilon^{-\beta}$ with $\beta \in(0, \alpha)$ are randomized on time scales that depend on $\beta$, but leave this issue for a further investigation.

The paper is organized as follows: in Section 2 we derive a representation of $\mathbb{E} \hat{\zeta}_{\varepsilon}(t, \xi)$ in terms of the average of the expectation of the exponential of some functional over Brownian paths, see Proposition 2.1. Section 3 is devoted to the presentation of the proofs of Theorems 1.1 and 1.2. Finally in Section 4 we give the proof of Theorem 1.3, and the short Section 5 contains the proof of a standard auxiliary result.

Acknowledgments. TC was supported by the NSF Career grant DMS-1151414, TK by the Polish National Science Center grant DEC-2012/07/B/ST1/03320, and LR by an AFOSR NSSEFF Fellowship and NSF grant DMS-1311903.

\section{A Brownian formula for the averaged wave function}

In this section, we consider the Schrödinger equation, without any assumption on the smallness of the random potential

$$
\begin{aligned}
& i \frac{\partial \psi}{\partial t}+\frac{1}{2} \Delta \psi-V(x) \psi=0 \\
& \psi(0, x)=\psi_{0}(x) .
\end{aligned}
$$

We assume that $\psi_{0} \in \mathcal{S}\left(\mathbb{R}^{d}\right)$ and $V(x)$ is a Gaussian, stationary random field with continuous realizations defined over a probability space $(\Omega, \mathcal{F}, \mathbb{P})$. The complex valued spectral measure $\hat{V}(d p)$ corresponding to the field

$$
V(x)=\frac{1}{(2 \pi)^{d}} \int_{\mathbb{R}^{d}} e^{i p \cdot x} \hat{V}(d p),
$$

has the covariance

$$
\mathbb{E}\left[\hat{V}(d p) \hat{V}^{*}(d q)\right]=(2 \pi)^{d} \delta(p-q) \hat{R}(p) d p d q, \quad p, q \in \mathbb{R}^{d}
$$


with a non-negative function $\hat{R} \in L^{1}\left(\mathbb{R}^{d}\right)$. As $V(x)$ is real valued and $\hat{R}(p) \geq 0$ for all $p \in \mathbb{R}^{d}$, we have $\hat{R}(-p)=\hat{R}(p)$, for all $p \in \mathbb{R}^{d}$. We do not assume in this section that $\hat{R}(p)$ has the Schonberg form (1.10) - this will be done starting with Section 3 onwards.

The goal of this section is to obtain a convenient representation for the average compensated wave function

$$
\bar{\zeta}(t, \xi)=\mathbb{E} \hat{\zeta}(t, \xi)
$$

with $\hat{\zeta}(t, \xi)$ defined in (1.15). Let us introduce

$$
E(t, z, p, \xi):=\exp \left\{z\left(\sqrt{i} B_{t}+t \xi\right) \cdot p\right\}, \quad(t, z, p, \xi) \in[0,+\infty) \times \mathbb{C} \times \mathbb{R}^{2 d},
$$

where $B_{t}$ is a $d$-dimensional standard Brownian motion over a probability space $(\Sigma, \mathcal{A}, \mathbb{Q})$. We denote by $\mathbb{M}$ the expectation w.r.t. $\mathbb{Q}$, and set

$$
c_{n}(t, \xi):=\mathbb{M}\left\{\int_{0}^{t} d s \int_{0}^{t} d s^{\prime} \int_{\mathbb{R}^{d}} E(s, p, i ; \xi) E\left(s^{\prime}, p,-i ; \xi\right) \hat{R}(p) d p\right\}^{n} .
$$

The following will be the starting point for our analysis of the asymptotic limits.

Proposition 2.1 We have

$$
\bar{\zeta}(t, \xi)=\hat{\psi}_{0}(\xi) \sum_{n=0}^{\infty} \frac{(-1)^{n}}{(2 n) ! !} c_{n}(t, \xi), \quad t \geq 0, \xi \in \mathbb{R}^{d}
$$

or equivalently

$$
\bar{\zeta}(t, \xi)=\hat{\psi}_{0}(\xi) \mathbb{M E}\left\{\exp \left\{\frac{i}{(2 \pi)^{d}} \int_{\mathbb{R}^{d}} \hat{V}(d p) \int_{0}^{t} E(s, i, p, \xi) d s\right\}\right\} .
$$

The rest of this section contains the proof of this proposition.

\subsection{The Duhamel expansion}

We re-write the Schrödinger equation (2.1) as an integral in time equation

$$
\hat{\psi}(t, \xi)=\hat{\psi}_{0}(\xi) e^{-i|\xi|^{2} t / 2}+\frac{1}{i} \int_{0}^{t} \int_{\mathbb{R}^{d}} \frac{\hat{V}\left(d p_{1}\right)}{(2 \pi)^{d}} \hat{\psi}\left(s_{1}, \xi-p_{1}\right) e^{-i|\xi|^{2}\left(t-s_{1}\right) / 2} d s_{1} .
$$

The compensated wave function $\hat{\zeta}(t, \xi)$ satisfies

$$
\hat{\zeta}(t, \xi)=\hat{\psi}_{0}(\xi)+\frac{1}{i} \int_{0}^{t} \int_{\mathbb{R}^{d}} \frac{\hat{V}\left(d p_{1}\right)}{(2 \pi)^{d}} \hat{\zeta}\left(s_{1}, \xi-p_{1}\right) \exp \left\{i\left(|\xi|^{2}-\left|\xi-p_{1}\right|^{2}\right) \frac{s_{1}}{2}\right\} d s_{1} .
$$


Iterating (2.8), we get an infinite series expansion for $\hat{\zeta}(t, \xi)$ :

$$
\hat{\zeta}(t, \xi)=\sum_{n=0}^{\infty} \hat{\zeta}_{n}(t, \xi)
$$

with $\hat{\zeta}_{0}(t, \xi)=\hat{\psi}_{0}(\xi)$, and the rest of the individual terms of the form

$$
\hat{\zeta}_{n}(t, \xi)=\left[\frac{1}{i(2 \pi)^{d}}\right]^{n} \int_{\Delta_{n}(t)} d s_{1, n} \int_{\mathbb{R}^{d n}} \hat{V}\left(d p_{1}\right) \ldots \hat{V}\left(d p_{n}\right) \hat{\psi}_{0}\left(\xi-\sum_{j=1}^{n} p_{j}\right) e^{i G_{n}}
$$

with the phase

$$
G_{n}=G_{n}\left(s_{1, n}, p_{1, n}\right)=\sum_{k=1}^{n}\left(\left|\xi-\sum_{j=1}^{k-1} p_{j}\right|^{2}-\left|\xi-\sum_{j=1}^{k} p_{j}\right|^{2}\right) \frac{s_{n-k}}{2} .
$$

Here, we denote $p_{1, n}:=\left(p_{1}, \ldots, p_{n}\right) \in \mathbb{R}^{n d}$, and $s_{1, n}:=\left(s_{1}, \ldots, s_{n}\right) \in \mathbb{R}^{n}$, so that $d s_{1, n}:=$ $d s_{1} d s_{2} \ldots d s_{n}$. We have also denoted in (2.10) by $\Delta_{n}(t)$ the time simplex

$$
\Delta_{n}(t)=\left\{\left(s_{1}, s_{2}, \ldots, s_{n}\right): 0 \leq s_{1} \leq s_{1} \leq \cdots \leq s_{n} \leq t\right\} .
$$

The next standard proposition shows that we may employ term-wise expectation. Its proof is standard, for the convenience of the reader, we present it in Section 5 ,

Proposition 2.2 (i) The series (2.9) for the function $\hat{\zeta}(t, \xi)$ converges almost surely for any initial data $\psi_{0} \in \mathcal{S}\left(\mathbb{R}^{d}\right)$.

(ii) For each $(t, \xi) \in \mathbb{R}^{1+d}$ fixed, we have

$$
\mathbb{E} \hat{\zeta}(t, \xi)=\sum_{n=0}^{\infty} \mathbb{E} \hat{\zeta}_{2 n}(t, \xi)
$$

(iii) Moreover, we have

$$
\mathbb{E}\|\hat{\zeta}(t, \cdot)\|_{L^{2}\left(\mathbb{R}^{d}\right)}^{2}=\left\|\hat{\psi}_{0}\right\|_{L^{2}\left(\mathbb{R}^{d}\right)}^{2}, \quad t \geq 0 .
$$

Note that when we introduce various scaling parameters, this proposition will not guarantee that the convergence will be uniform in those parameters. Thus, while this proposition allows us to interchange the expectation and the summation of the series, it will not allow us to pass to the limit in the individual terms of the series when we will consider appropriate asymptotic limits.

Using Proposition 2.2, and the rule of the expectation for the product of $2 n$ mean zero Gaussian random variables, we may re-write the expectation as a series

$$
\bar{\zeta}(t, \xi):=\mathbb{E} \zeta(t, \xi)=\left\{1+\sum_{n=1}^{+\infty} \sum_{\mathcal{F} \in \mathfrak{F}_{2 n}} I(\mathcal{F})(t)\right\} \hat{\psi}_{0}(\xi) .
$$


Here, $\mathfrak{F}_{2 n}$ is the collection of all pairings $\mathcal{F}$ formed over the set $\mathbb{Z}_{2 n}:=\{1, \ldots, 2 n\}$. The individual terms for each pairing have the form

$$
I(\mathcal{F}):=(-1)^{n} \int_{\mathbb{R}^{2 d n}} \prod_{(k, \ell) \in \mathcal{F}} \delta\left(p_{k}+p_{\ell}\right) \hat{R}\left(p_{k}\right) d p_{1,2 n} \int_{\Delta_{2 n}(t)} e^{i G_{2 n}} d s_{1,2 n}
$$

where $d p_{1, n}=d p_{1} \ldots d p_{n}$, and

$$
G_{2 n}\left(s_{1,2 n}, p_{1,2 n}\right)=\sum_{m=1}^{2 n}\left(s_{2 n-m+1}-s_{2 n-m}\right)\left(\xi \cdot \sum_{j=1}^{m} p_{j}-\frac{1}{2}\left|\sum_{j=1}^{m} p_{j}\right|^{2}\right),
$$

where $s_{0}:=0$.

\subsection{The proof of Proposition 2.1}

We now prove Proposition 2.1 via an alternative representation for the series (2.14) for $\bar{\zeta}(t, \xi)$. Identity (2.5) can be restated as

$$
\sum_{\mathcal{F} \in \widetilde{F}_{2 n}} I(\mathcal{F})(t)=\frac{(-1)^{n} c_{n}(t, \xi)}{(2 n) ! !}, \quad \forall n \geq 1 .
$$

We first find an expression for the left side of (2.17). Given a pairing $\mathcal{F}$, and $m \in\{1, \ldots, 2 n\}$, let us denote by $A_{m}(\mathcal{F})$ the set of all left vertices $\ell \leq m$ such that the corresponding right vertex $r$ satisfies $r>m$, that is, the edge $(l r)$ crosses over $m$, with the convention $A_{0}(\mathcal{F}):=\emptyset$. Sometimes, when the pairing is obvious from the context, we simply write $A_{m}$. We also denote by $\mathcal{L}(\mathcal{F})$ the set of all left vertices of $\mathcal{F}$. For a given pairing $\mathcal{F}$, we have

$$
\sum_{j=1}^{k} p_{j}=\sum_{j \in A_{k}} p_{j}
$$

a.e. in the measure

$$
\prod_{(k, \ell) \in \mathcal{F}} \delta\left(p_{k}+p_{\ell}\right) \hat{R}\left(p_{k}\right) d p_{1,2 n}
$$

The phase $G_{2 n}$ has, therefore, the form (note that the set $A_{2 n}$ is empty)

$$
G_{2 n}\left(s_{1,2 n}, p_{1,2 n}\right)=\sum_{m=1}^{2 n-1}\left(s_{2 n-m+1}-s_{2 n-m}\right)\left(\xi \cdot \sum_{j \in A_{m}} p_{j}-\frac{1}{2}\left|\sum_{j \in A_{m}} p_{j}\right|^{2}\right) .
$$

This leads to the expression

$$
I(\mathcal{F})(t)=(-1)^{n} \int_{\Delta_{2 n}(t)} d s_{1,2 n} \int_{\mathbb{R}^{n d}}\left[\prod_{\ell \in \mathcal{L}(\mathcal{F})} \hat{R}\left(p_{\ell}\right)\right]
$$




$$
\times \exp \left\{\sum_{m=1}^{2 n-1}\left(s_{2 n-m+1}-s_{2 n-m}\right)\left(-\frac{i}{2}\left|\sum_{j \in A_{m}(\mathcal{F})} p_{j}\right|^{2}+i \xi \cdot \sum_{j \in A_{m}(\mathcal{F})} p_{j}\right)\right\} \prod_{\ell \in \mathcal{L}(\mathcal{F})} d p_{\ell} .
$$

Next, we re-write $c_{n}$ defined by (2.4), to make it clear that (2.17) holds. To abbreviate somewhat the notation, we will denote $E(s, i, p, \xi)$ by $E(s, p)$ (see (2.3) ). We can re-write $c_{n}(t, \xi)$, see (2.4), as

$$
c_{n}=\mathbb{M}\left\{\int_{0}^{t} \ldots \int_{0}^{t} d s_{1,2 n} \int_{\mathbb{R}^{2 n d}} d p_{1,2 n} \prod_{j=1}^{n} \hat{R}\left(p_{2 j-1}\right) \delta\left(p_{2 j-1}+p_{2 j}\right) \prod_{j=1}^{2 n} E\left(s_{j}, p_{j}\right)\right\} .
$$

For each $\left(s_{1}, s_{2}, \ldots, s_{2 n}\right)$, we re-order the times $s_{j}$ in the increasing order, and re-label the indices $j$ accordingly, so that

$$
c_{n}=\sum_{\sigma} \int_{\Delta_{2 n}(t)} d s_{1,2 n} \int_{\mathbb{R}^{2 n d}} d p_{1,2 n} \prod_{j=1}^{n}\left[\hat{R}\left(p_{\sigma(2 j-1)}\right) \delta\left(p_{\sigma(2 j-1)}+p_{\sigma(2 j)}\right)\right] \mathbb{M}\left\{\prod_{j=1}^{2 n} E\left(s_{\sigma(j)}, p_{\sigma(j)}\right)\right\} .
$$

Here, the summation extends over all possible permutations $\sigma:\{1, \ldots, 2 n\} \rightarrow\{1, \ldots, 2 n\}$. The symmetry of the last product above allows us to write

$$
c_{n}=\sum_{\sigma} \int_{\Delta_{2 n}(t)} d s_{1,2 n} \int_{\mathbb{R}^{2 n d}} d p_{1,2 n} \prod_{j=1}^{n}\left[\hat{R}\left(p_{\sigma(2 j-1)}\right) \delta\left(p_{\sigma(2 j-1)}+p_{\sigma(2 j)}\right)\right] \mathbb{M}\left\{\prod_{j=1}^{2 n} E\left(s_{j}, p_{j}\right)\right\} .
$$

Using the independence of the increments of a Brownian motion, and performing the expectation we conclude that (with $s_{2 n+1}=0$ )

$$
c_{n}=\sum_{\sigma} \int_{\Delta_{2 n}(t)} d s_{1,2 n} \int_{\mathbb{R}^{2 n d}} d p_{1,2 n} \prod_{j=1}^{n}\left[\hat{R}\left(p_{\sigma(2 j-1)}\right) \delta\left(p_{\sigma(2 j-1)}+p_{\sigma(2 j)}\right)\right] S_{n}\left(s_{1,2 n}, p_{1,2 n}\right),
$$

where

$$
S_{n}\left(s_{1,2 n}, p_{1,2 n}\right):=\prod_{j=1}^{2 n} \exp \left\{\left(s_{2 n-j+1}-s_{2 n-j}\right)\left(-\frac{i}{2}\left|\sum_{m=1}^{j} p_{m}\right|^{2}+i \xi \cdot \sum_{m=1}^{j} p_{m}\right)\right\} .
$$

Note that (2.19) and (2.21) are very similar, making (2.17) "plausible", except for the summation taken over all permutations $\sigma$ in (2.21), as opposed to the summation over all pairings $\mathcal{F}$ in the left side of (2.17). As we will see, the difference in the two summations is responsible for the factor $1 /(2 n) !$ ! in (2.17).

To reconcile the two summations, let $\Pi(2 n)$ be the set of all permutations of $\{1, \ldots, 2 n\}$, and define the mapping $\mathfrak{f}: \Pi(2 n) \rightarrow \mathfrak{F}_{2 n}$ as follows. Given a permutation $\sigma$, we let $\mathfrak{f}(\sigma)$ be the following pairing: a pair $(\ell, r)$, with $\ell<r$ is in $\mathfrak{f}(\sigma)$ iff there exists $j$ such that $\ell=\sigma(2 j-1)$ 
and $r=\sigma(2 j)$, or $\ell=\sigma(2 j)$ and $r=\sigma(2 j-1)$. In other words, we start with the simple pairing $(1,2)(3,4) \ldots(2 n-1,2 n)$ and map it by $\sigma$ to the pairing

$$
(\sigma(1), \sigma(2))(\sigma(3), \sigma(4)) \ldots(\sigma(2 n-1), \sigma(2 n)),
$$

with a slight abuse of notation, as it is possible that $\sigma(2 j-1)>\sigma(2 j)$. Observe that if $\mathcal{F}=\mathfrak{f}(\sigma)$ then

$$
\begin{aligned}
& \int_{\mathbb{R}^{2 n d}} S_{n}\left(s_{1,2 n}, p_{1,2 n}\right) \prod_{j=1}^{n}\left[\hat{R}\left(p_{\sigma(2 j-1)}\right) \delta\left(p_{\sigma(2 j-1)}+p_{\sigma(2 j)}\right)\right] d p_{1,2 n} \\
& =\int_{\mathbb{R}^{n d}} S_{n}\left(s_{1,2 n}, p_{1,2 n}\right)\left[\prod_{\ell \in \mathcal{L}(\mathcal{F})} \hat{R}\left(p_{\ell}\right) d p_{\ell}\right] .
\end{aligned}
$$

On the other hand, given a pairing $\mathcal{F} \in \mathfrak{F}_{2 n}$ :

$$
\mathcal{F}:=\left\{\left(\ell_{1}, r_{1}\right), \ldots,\left(\ell_{n}, r_{n}\right)\right\}
$$

with $1=\ell_{1}<\ell_{2}<\ldots<\ell_{n}$, we may define the corresponding permutation $\mathfrak{g}(\mathcal{F}) \in \Pi(2 n)$ as $\left(\ell_{1}, r_{1}, \ell_{2}, r_{2}, \ldots, \ell_{n}, r_{n}\right)$. This defines the mapping $\mathfrak{g}: \mathfrak{F}_{2 n} \rightarrow \Pi(2 n)$ such that

$$
\mathfrak{f}(\mathfrak{g}(\mathcal{F}))=\mathcal{F}, \quad \forall \mathcal{F} \in \mathfrak{F}_{2 n}
$$

Thus, the mapping $\mathfrak{f}$ is onto. Next, suppose that $\sigma=\mathfrak{g}(\mathcal{F})$ and $\mathcal{F}$ is given by (2.24). Note that any permutation $\sigma^{\prime}$ obtained from $\sigma$ by a transposition of $\ell_{j}$ and $r_{j}$, as well as by permuting in the same fashion $\ell_{1}, \ldots \ell_{n}$ and $r_{1}, \ldots, r_{n}$, satisfies $\mathfrak{f}\left(\sigma^{\prime}\right)=\mathcal{F}$. For each permutation $\sigma=\mathfrak{g}(\mathcal{F})$ there exist $2^{n} n !=(2 n)$ !! different permutations obtained in that way. Writing $\mathcal{F}_{\sigma}=\mathfrak{f}(\sigma)$ we obtain from (2.21) and (2.23)

$$
\begin{aligned}
& \frac{c_{n}}{(2 n) ! !}=\frac{1}{(2 n) ! !} \int_{\Delta_{2 n}(t)} d s_{1,2 n} \int_{\mathbb{R}^{2 n d}} S_{n}\left(s_{1,2 n}, p_{1,2 n}\right) \prod_{j=1}^{n}\left[\hat{R}\left(p_{\sigma(2 j-1)}\right) \delta\left(p_{\sigma(2 j-1)}+p_{\sigma(2 j)}\right)\right] d p_{1,2 n} \\
& \quad=\frac{1}{(2 n) ! !} \sum_{\sigma} \int_{\Delta_{2 n}(t)} d s_{1,2 n} \int\left[\prod_{\ell \in \mathcal{L}\left(\mathcal{F}_{\sigma}\right)} S_{n}\left(s_{1,2 n}, p_{1,2 n}\right) \hat{R}\left(p_{\ell}\right) d p_{\ell}\right] \\
& \quad=\sum_{\mathcal{F}} \int_{\Delta_{2 n}(t)} d s_{1,2 n} \int\left[\prod_{\ell \in \mathcal{L}(\mathcal{F})} S_{n}\left(s_{1,2 n}, p_{1,2 n}\right) \hat{R}\left(p_{\ell}\right) d p_{\ell}\right] .
\end{aligned}
$$

Comparing to (2.19), we conclude that (2.17) holds, finishing the proof of (2.17) and thus also of Proposition 2.1. 


\section{The finite effective potential regime}

In this section, we present the proofs of Theorems 1.1 and 1.2, both of which hold when the effective potential $R_{*}<+\infty$, that is, $\gamma>3-d$, and the non-trivial behavior takes place at times of the order $t \sim \varepsilon^{-2}$.

Let us add the weak coupling limit to the representation in Proposition 2.1. Recall that, the wave function $\psi^{(\varepsilon)}$ is the solution of

$$
\begin{aligned}
& i \frac{\partial \psi^{(\varepsilon)}}{\partial t}+\frac{1}{2} \Delta \psi^{(\varepsilon)}-\varepsilon V(x) \psi^{(\varepsilon)}=0, \\
& \psi^{(\varepsilon)}(0, x)=\varepsilon^{d \beta / 2} \psi_{0}\left(\varepsilon^{\beta} x\right)
\end{aligned}
$$

with $\beta \geq 0$, and the compensated wave function is given by (1.21). We may now apply Proposition 2.1. replacing the random potential $V \rightarrow \varepsilon V$, and the time $t \rightarrow t / \varepsilon^{2}$ in (2.4) and (2.6) . Using, in addition, the representation (1.10) for the power spectrum $\hat{R}(p)$ turns (2.5) when $\beta=0$, into

$$
\bar{\zeta}_{\varepsilon}(t, \xi):=\mathbb{E} \hat{\zeta}_{\varepsilon}(t, \xi)=\hat{\psi}_{0}(\xi) \sum_{n=0}^{+\infty} \frac{(-1)^{n} c_{n, \varepsilon}(t, \xi)}{(2 n) ! !}, \quad \forall(t, \xi) \in \mathbb{R}^{1+d},
$$

where

$$
c_{n, \varepsilon}(t, \xi):=\varepsilon^{2 n} \mathbb{M}\left\{\int_{1}^{+\infty} \frac{s(\lambda) d \lambda}{\lambda^{\gamma}} \int_{0}^{t / \varepsilon^{2}} d s \int_{0}^{t / \varepsilon^{2}} d s^{\prime} \int_{\mathbb{R}^{d}} E(s, i, p, \xi) E\left(s^{\prime},-i, p, \xi\right) e^{-\lambda^{2}|p|^{2} / 2} d p\right\}^{n}
$$

and $E(s, i, p, \xi)$ is given by (2.3) . On the other hand, when $\beta>0$, we have

$$
\bar{\zeta}_{\varepsilon}(t, \xi)=\hat{\psi}_{0}(\xi) \sum_{n=0}^{+\infty} \frac{(-1)^{n}}{(2 n) ! !} c_{n, \varepsilon}\left(t, \varepsilon^{\beta} \xi\right)
$$

\section{A formal analysis for $\xi=0$}

Before we embark on the proof of the main results, we note that, say, when $\beta=0$, we may use the definition (2.3) of $E(s, i, p, \xi)$ to re-sum the series (3.2). This gives

$$
\begin{aligned}
\bar{\zeta}_{\varepsilon}(t, \xi)= & \hat{\psi}_{0}(\xi) \mathbb{M}\left\{\operatorname { e x p } \left\{-\frac{\varepsilon^{2}}{2} \int_{1}^{+\infty} \frac{s(\lambda) d \lambda}{\lambda^{\gamma}} \int_{0}^{t / \varepsilon^{2}} d s \int_{0}^{t / \varepsilon^{2}} d s^{\prime}\right.\right. \\
& \left.\left.\times \int_{\mathbb{R}^{d}} e^{-\lambda^{2}|p|^{2} / 2} \exp \left\{i\left(B_{s}^{(\xi)}-B_{s^{\prime}}^{(\xi)}\right) \cdot p\right\}\right\} d p\right\}
\end{aligned}
$$

with

$$
B_{s}^{(\xi)}:=\sqrt{i} B_{s}+\xi s
$$

Performing the integration over the $p$ variable we obtain the following representation of the averaged compensated wave function when $\beta=0$ (the microscopic initial condition). 
Proposition 3.1 Suppose that $\psi_{0} \in \mathcal{S}\left(\mathbb{R}^{d}\right)$, then

$$
\bar{\zeta}_{\varepsilon}(t, \xi)=\hat{\psi}_{0}(\xi) \mathbb{M} \exp \left\{-\frac{1}{(2 \pi)^{d / 2}} \int_{1}^{+\infty} Z_{\varepsilon}(t, \lambda, \xi) \frac{s(\lambda) d \lambda}{\lambda^{\gamma+d}}\right\} .
$$

Here

$$
Z_{\varepsilon}(t, \lambda, \xi):=\frac{\varepsilon^{2}}{2} \int_{0}^{t / \varepsilon^{2}} d s \int_{0}^{t / \varepsilon^{2}} d s^{\prime} \exp \left\{-\frac{1}{2 \lambda^{2}}\left(B_{s}^{(\xi)}-B_{s^{\prime}}^{(\xi)}\right)^{2}\right\}
$$

and $a^{2}:=\sum_{j=1}^{d} a_{j}^{2}$ for any $a=\left(a_{1}, \ldots, a_{d}\right) \in \mathbb{C}^{d}$.

It is straightforward to write down the analog for $\beta>0$ by replacing $\xi$ by $\varepsilon^{\beta} \xi$ in (3.1) and (3.8).

Representation (3.7) is quite simple and elegant, and seems a natural starting point for the proof of the main results of this paper. However, because of the double exponential appearing in (3.7) and (3.8), we were unable to find a simple way to pass to the limit $\varepsilon \rightarrow 0+$ in (3.7). As we show below, one can relatively easily pass to the limit in (3.8), at least when $\xi=0$, and obtain a limit for $Z_{\varepsilon}(t, \lambda, 0)$, but even for $\xi=0$ the justification of interchanging the limit and the expectation in (3.7) eluded us. This strategy for $\xi \neq 0$ seems not to be simpler than what is done in the rest of the paper, and the justification of interchanging the limit and the expectation seems non-trivial, so we do not pursue this route here.

We now describe how one passes to the limit in (3.8) in the simplest case $\xi=0$, as this gives a relatively quick formal way to the general answer. We will show that $Z_{\varepsilon}(t, \lambda):=Z_{\varepsilon}(t, \lambda, 0)$ has a deterministic limit as $\varepsilon \rightarrow 0$ :

$$
Z_{\varepsilon}(t, \lambda) \rightarrow \bar{Z}(t, \lambda):=-\frac{2 i \lambda^{2} t}{d-2} .
$$

Recall that our analysis holds in dimension $d>2$. This is in agreement with (1.19), as

$$
r(0)=\frac{2}{(2 \pi)^{d / 2}(d-2)} \int_{1}^{\infty} \frac{s(\lambda) d \lambda}{\lambda^{\gamma+d-2}} .
$$

To show (3.9), first note that

$$
\begin{aligned}
\mathbb{M} Z_{\varepsilon}(t, \lambda) & =\varepsilon^{2} \int_{0}^{t / \varepsilon^{2}} d s \int_{0}^{s} \mathbb{M} \exp \left\{-\frac{i\left|B_{s^{\prime}}\right|^{2}}{2 \lambda^{2}}\right\} d s^{\prime} \\
& =\frac{\varepsilon^{2}}{(2 \pi)^{d / 2}} \int_{0}^{t / \varepsilon^{2}} d s \int_{0}^{s} d s^{\prime} \int_{\mathbb{R}^{d}} \exp \left\{-\frac{|y|^{2}}{2}\left(1+\frac{i s^{\prime}}{\lambda^{2}}\right)\right\} d y \\
& =\varepsilon^{2} \lambda^{2} \int_{0}^{t / \varepsilon^{2}} d s \int_{0}^{s / \lambda^{2}} \frac{d s^{\prime}}{\left(1+i s^{\prime}\right)^{d / 2}}=\varepsilon^{2} \lambda^{2} \int_{0}^{t /\left(\lambda^{2} \varepsilon^{2}\right)}\left(\frac{t}{\varepsilon^{2}}-\lambda^{2} s^{\prime}\right) \frac{d s^{\prime}}{\left(1+i s^{\prime}\right)^{d / 2}} \\
& \rightarrow \lambda^{2} t \int_{0}^{\infty} \frac{d s^{\prime}}{\left(1+i s^{\prime}\right)^{d / 2}}=-\frac{2 i \lambda^{2} t}{d-2}
\end{aligned}
$$


so that

$$
\mathbb{M} Z_{\varepsilon}(t, \lambda) \rightarrow \bar{Z}(t, \lambda)
$$

given by (3.9).

Next, we look at the second moment

$$
\mathbb{M}\left|Z_{\varepsilon}(t, \lambda)\right|^{2}=\frac{\varepsilon^{4}}{4} \int_{0}^{t / \varepsilon^{2}} \cdots \int_{0}^{t / \varepsilon^{2}} \mathbb{M} \exp \left\{-\frac{i}{2 \lambda^{2}}\left[\left|B_{s_{1}}-B_{s_{2}}\right|^{2}-\left|B_{s_{3}}-B_{s_{4}}\right|^{2}\right]\right\} d s_{1,4} .
$$

In the ensuing calculation we show that

$$
\lim _{\varepsilon \rightarrow 0+} \mathbb{M}\left|Z_{\varepsilon}(t, \lambda)\right|^{2}=\bar{Z}(t, \lambda)^{2},
$$

which, combined with (3.12), proves that

$$
\lim _{\varepsilon \rightarrow 0+} Z_{\varepsilon}(t, \lambda)=\bar{Z}(t, \lambda)
$$

in the $L^{2}$ sense, with respect to the randomness. As we have mentioned, this argument does not allow us to exchange the limit, as $\varepsilon \rightarrow 0+$, and the expectation in (3.7). If we formally do this, using (3.15), we conclude that (1.19) holds for $\xi=0$. Making this argument fully rigorous, including for $\xi \neq 0$, is essentially what is done in the rest of this paper

To show that (3.14) holds, we follow the argument made in Section 2.2. We may use (3.13) to write

$$
\mathbb{M}\left|Z_{\varepsilon}(t, \lambda)\right|^{2}=I_{1, \varepsilon}+I_{2, \varepsilon}+I_{3, \varepsilon}
$$

with

$$
\begin{aligned}
& I_{1, \varepsilon}:=2 \varepsilon^{4} \int_{\Delta_{4}\left(t / \varepsilon^{2}\right)} \mathbb{M} \exp \left\{-\frac{i}{2 \lambda^{2}}\left[\left|B_{s_{1}}-B_{s_{2}}\right|^{2}-\left|B_{s_{3}}-B_{s_{4}}\right|^{2}\right]\right\} d s_{1,4}, \\
& I_{2, \varepsilon}:=2 \varepsilon^{4} \int_{\Delta_{4}\left(t / \varepsilon^{2}\right)} \mathbb{M} \exp \left\{-\frac{i}{2 \lambda^{2}}\left[\left|B_{s_{1}}-B_{s_{3}}\right|^{2}-\left|B_{s_{2}}-B_{s_{4}}\right|^{2}\right]\right\} d s_{1,4}, \\
& I_{3, \varepsilon}:=2 \varepsilon^{4} \int_{\Delta_{4}\left(t / \varepsilon^{2}\right)} \mathbb{M} \exp \left\{-\frac{i}{2 \lambda^{2}}\left[\left|B_{s_{1}}-B_{s_{4}}\right|^{2}-\left|B_{s_{2}}-B_{s_{3}}\right|^{2}\right]\right\} d s_{1,4},
\end{aligned}
$$

where $\Delta_{4}\left(t / \varepsilon^{2}\right):=\left[t / \varepsilon^{2} \geq s_{4} \geq \ldots \geq s_{1} \geq 0\right]$ and $d s_{1,4}:=d s_{1} d s_{2} d s_{3} d s_{4}$. Let

$$
\Delta_{4}^{\prime}\left(t / \varepsilon^{2}\right):=\left[\sum_{j=1}^{4} \tau_{j} \leq t / \varepsilon^{2}, \tau_{j} \geq 0, j=1, \ldots, 4\right] .
$$

A direct calculation, as in the case of the first moment of $Z_{\varepsilon}(t, \lambda)$, shows that

$$
I_{1, \varepsilon}=2(\varepsilon \lambda)^{4} \int_{\Delta_{4}^{\prime}\left(t / \varepsilon^{2}\right)} d \tau_{1,4} \prod_{j=1}^{2} \frac{1}{\left(1+(-1)^{j} i \tau_{2 j}\right)^{d / 2}} \rightarrow(\bar{Z}(t, \lambda))^{2},
$$


as $\varepsilon \rightarrow 0$. Then, (3.14) follows, provided we show that

$$
\lim _{\varepsilon \rightarrow 0+} I_{j, \varepsilon}=0, \quad j=2,3
$$

Note that

$$
I_{2, \varepsilon}=\frac{2 \varepsilon^{4}}{(2 \pi)^{3 d / 2}} \int_{\Delta_{4}^{\prime}\left(t / \varepsilon^{2}\right)} d \tau_{1,4} \int_{\mathbb{R}^{3 d}} \exp \left\{-\frac{1}{2}\left(\left(I_{3 d}+i D\right) y, y\right)_{\mathbb{R}^{3 d}}\right\} d y_{1,3},
$$

with $y:=\left[y_{1}, y_{2}, y_{3}\right], I_{d}$ the $d \times d$ identity matrix and $D$ the $3 d \times 3 d$ block matrix obtained from

$$
D_{r}=\left[\begin{array}{ccc}
\frac{\tau_{2}}{\lambda^{2}} & \frac{\left(\tau_{2} \tau_{3}\right)^{1 / 2}}{\lambda^{2}} & 0 \\
\frac{\left(\tau_{2} \tau_{3}\right)^{1 / 2}}{\lambda^{2}} & 0 & -\frac{\left(\tau_{3} \tau_{4}\right)^{1 / 2}}{\lambda^{2}} \\
0 & -\frac{\left(\tau_{3} \tau_{4}\right)^{1 / 2}}{\lambda^{2}} & -\frac{\tau_{4}}{\lambda^{2}}
\end{array}\right]
$$

by replacing each entry of $D_{r}$ by the respective $d \times d$ diagonal block. To evaluate the $y$-integral in (3.19) we use the following elementary result.

Lemma 3.2 Suppose that $f: \mathbb{C}^{N} \rightarrow \mathbb{C}$ is a holomorphic function such that there exists $C>0$, for which

$$
\left|f\left(z_{1}, \ldots, z_{N}\right)\right| \leq C \exp \left\{C \sum_{j=1}^{N}\left|z_{j}\right|\right\}, \quad\left(z_{1}, \ldots, z_{N}\right) \in \mathbb{C}^{N}
$$

and $A$ is a symmetric $N \times N$-matrix with eigenvalues $\lambda_{1} \geq \lambda_{2} \geq \ldots \geq \lambda_{N}$. Then,

$$
\begin{aligned}
\int_{\mathbb{R}^{N}} \exp & \left\{-\frac{1}{2}\left(\left(I_{N}+z A\right) x, x\right)_{\mathbb{R}^{N}}\right\} f(x) d x \\
& =\left[\operatorname{det}\left(I_{N}+z A\right)\right]^{-1 / 2} \int_{\mathbb{R}^{N}} \exp \left\{-\frac{|x|^{2}}{2}\right\} f\left(\left(I_{N}+z A\right)^{-1 / 2} x\right) d x,
\end{aligned}
$$

for all $z \in \mathbb{C}$ such that $\operatorname{Re}\left(1+z \lambda_{j}\right)>0, j=1, \ldots, N$.

The formula (3.21) obviously holds for $z$ real. It can be extended to the set in question by the analytic continuation argument.

Using the above lemma we conclude that

$$
I_{2, \varepsilon}=2 \varepsilon^{4} \int_{\Delta_{4}^{\prime}\left(t / \varepsilon^{2}\right)} \operatorname{det}\left(I_{3}+i D_{r}\right)^{-d / 2} d \tau_{1,4} .
$$

A direct calculation yields

$$
\operatorname{det}\left(I_{3}+i D_{r}\right)=1+\frac{\tau_{2} \tau_{4}+\tau_{3} \tau_{2}+\tau_{3} \tau_{4}}{\lambda^{4}}+\frac{i\left(\tau_{2}-\tau_{4}\right)}{\lambda^{2}} .
$$


Hence, we have

$$
\left|I_{2, \varepsilon}\right| \leq C \varepsilon^{4} \int_{\Delta_{4}^{\prime}\left(t / \varepsilon^{2}\right)}\left\{1+\frac{\tau_{2} \tau_{4}+\tau_{3} \tau_{2}+\tau_{3} \tau_{4}}{\lambda^{4}}+\frac{\left|\tau_{2}-\tau_{4}\right|}{\lambda^{2}}\right\}^{-d / 2} d \tau_{1,4} .
$$

Changing variables $\tau_{j}^{\prime}:=\varepsilon^{2} \tau_{j}, j=1,3$, we obtain

$$
\left|I_{2, \varepsilon}\right| \leq 2^{d / 2+1} \int_{\Delta_{2}^{\prime}(t)} d \tau_{1} d \tau_{3} \int_{0}^{+\infty} \int_{0}^{+\infty}\left\{1+\frac{\varepsilon^{-2} \tau_{3}\left(\tau_{2}+\tau_{4}\right)+\tau_{2} \tau_{4}}{\lambda^{4}}+\frac{\left|\tau_{2}-\tau_{4}\right|}{\lambda^{2}}\right\}^{-d / 2} d \tau_{2} d \tau_{4} \rightarrow 0
$$

as $\varepsilon \rightarrow 0+$, since the integrand is bounded by the function $\left\{1+\tau_{2} \tau_{4} \lambda^{-4}+\left|\tau_{2}-\tau_{4}\right| \lambda^{-2}\right\}^{-d / 2}$ that is absolutely integrable in $[0,+\infty)^{2}$ for $d \geq 3$. Similarly, we have

$$
I_{3, \varepsilon}=2 \varepsilon^{4} \int_{\Delta_{4}^{\prime}\left(t / \varepsilon^{2}\right)} \operatorname{det}\left(I_{3}+i E_{r}\right)^{-d / 2} d \tau_{1,4}
$$

with

One can easily obtain

$$
E_{r}=\left[\begin{array}{ccc}
\frac{\tau_{2}}{\lambda^{2}} & \frac{\left(\tau_{2} \tau_{3}\right)^{1 / 2}}{\lambda^{2}} & \frac{\left(\tau_{2} \tau_{4}\right)^{1 / 2}}{\lambda^{2}} \\
\frac{\left(\tau_{2} \tau_{3}\right)^{1 / 2}}{\lambda^{2}} & 0 & \frac{\left(\tau_{3} \tau_{4}\right)^{1 / 2}}{\lambda^{2}} \\
\frac{\left(\tau_{2} \tau_{4}\right)^{1 / 2}}{\lambda^{2}} & \frac{\left(\tau_{3} \tau_{4}\right)^{1 / 2}}{\lambda^{2}} & \frac{\tau_{4}}{\lambda^{2}}
\end{array}\right]
$$

$$
\operatorname{det}\left(I_{3}+i E_{r}\right)=1+\frac{\tau_{2} \tau_{3}+\tau_{3} \tau_{4}}{\lambda^{4}}+\frac{i\left(\tau_{2}+\tau_{4}\right)}{\lambda^{2}}
$$

It follows that

$$
\left|I_{3, \varepsilon}\right| \leq 2^{d / 2+1} \varepsilon^{4} \int_{\Delta_{4}^{\prime}\left(t / \varepsilon^{2}\right)}\left\{1+\frac{\tau_{3} \tau_{2}+\tau_{3} \tau_{4}}{\lambda^{4}}+\frac{\tau_{2}+\tau_{4}}{\lambda^{2}}\right\}^{-d / 2} d \tau_{1,4}
$$

Changing variables $\tau_{j}^{\prime}:=\varepsilon^{2} \tau_{j}, j=1,2$ we conclude that

$$
\left|I_{3, \varepsilon}\right| \leq C \int_{\Delta_{2}^{\prime}(t)} d \tau_{1} d \tau_{2} \int_{0}^{t / \varepsilon^{2}} d \tau_{3}\left(\int_{0}^{t / \varepsilon^{2}}\left\{A_{\varepsilon}+B \tau_{4}\right\}^{-d / 2} d \tau_{4}\right)
$$

with

$$
A_{\varepsilon}:=1+\left(\lambda^{2} \varepsilon\right)^{-2} \tau_{3} \tau_{2}+(\lambda \varepsilon)^{-2} \tau_{2}, \quad B:=\frac{\tau_{3}}{\lambda^{4}}+\frac{1}{\lambda^{2}} .
$$

Substituting $\tau_{4}^{\prime}:=A_{\varepsilon}+B \tau_{4}$ in the last integral we obtain

$$
\left|I_{3, \varepsilon}\right| \leq C \int_{\Delta_{2}^{\prime}(t)} d \tau_{1} d \tau_{2} \int_{0}^{t / \varepsilon^{2}} B^{-1} A_{\varepsilon}^{1-d / 2} d \tau_{3}
$$




$$
\leq C \lambda^{4} \log \left[\frac{t}{(\varepsilon \lambda)^{2}}+1\right] \int_{\Delta_{2}^{\prime}(t)}\left(1+(\lambda \varepsilon)^{-2} \tau_{2}\right)^{1-d / 2} d \tau_{1} d \tau_{2} \rightarrow 0
$$

as $\varepsilon \rightarrow 0+$, since $d>2$. Thus, (3.18) follows, and the proof of (3.15) is complete. As we have mentioned, unfortunately, the passage to the limit in (3.8) for $\xi \neq 0$ and justification of the interchange of the limit and expectation in (3.7) do not seem simpler than what is done in the rest of the paper, so we do not use (3.7) and (3.8) below.

\section{A uniform bound on $c_{n, \varepsilon}(t, \xi)$}

The main step in the proof of Theorems 1.1 and 1.2 is the following uniform bound on $c_{n, \varepsilon}$ that allows us to pass to the limit in representations (3.2) and (3.4).

Proposition 3.3 Suppose that $d \geq 3$ and $\gamma>3-d$. Then, there exists $C>0$ such that for all $n \geq 0, \varepsilon \in(0,1], \xi \in \mathbb{R}^{d}$ and $t \geq 0$ we have

$$
\left|c_{n, \varepsilon}(t, \xi)\right| \leq n !(C t)^{n}
$$

\section{An alternative representation for $c_{n, \varepsilon}(t, \xi)$}

Both in the proof of Proposition 3.3, and in passing to the limit $\varepsilon \rightarrow 0$ in $c_{n, \varepsilon}(t, \xi)$, it will be convenient for us to use an expression different from (3.3). Let us first introduce some notation. Given a permutation $\sigma$ of $\{1, \ldots, 2 n\}$, we have the corresponding pairing in $\mathfrak{F}_{2 n}$ defined by

$$
\mathfrak{f}(\sigma)=\left\{\left(\ell_{1}, r_{1}\right), \ldots,\left(\ell_{n}, r_{n}\right)\right\}
$$

with

$$
\left(\ell_{k}, r_{k}\right):= \begin{cases}(\sigma(2 k-1), \sigma(2 k)), & \text { if } \sigma(2 k-1)<\sigma(2 k), \\ (\sigma(2 k), \sigma(2 k-1)), & \text { if } \sigma(2 k)<\sigma(2 k-1) .\end{cases}
$$

We may then define a $2 n d \times 2 n d$ symmetric non-negative matrix $A_{\sigma}(\tau, \lambda)$ corresponding to the quadratic form

$$
\Phi_{\sigma}(y)=\left(A_{\sigma}(\tau, \lambda) y, y\right)_{\mathbb{R}^{2 d n}}=\sum_{k=1}^{n} \frac{1}{\lambda_{r_{k}}^{2}}\left|\sum_{j=\ell_{k}+1}^{r_{k}} y_{j} \tau_{j}^{1 / 2}\right|^{2}
$$

for $y=\left(y_{1}, \ldots, y_{2 n}\right) \in \mathbb{R}^{2 d n}$ and $\tau_{j}:=s_{j}-s_{j-1}, j=1, \ldots, 2 n$ with $s_{0}:=0$. In order to describe the matrix $A_{\sigma}(\tau, \lambda)$ more explicitly, we introduce some terminology.

For each $j \in\{2, \ldots, 2 n\}$ define

$$
a_{j, j}:=\sum_{k}^{\prime} \frac{1}{\lambda_{r_{k}}^{2}}
$$


where the summation extends over those $k$-s, for which $\ell_{k}<j \leq r_{k}$. Given $m<j$ we let

$$
a_{m, j}=a_{j, m}=\sum_{k} \frac{1}{\lambda_{r_{k}}^{2}}
$$

with the summation extending over those $k$-s, for which $\ell_{k}<m<j \leq r_{k}$. We also let

$$
a_{1, j}=a_{j, 1}=0 \quad \text { for all } \quad j \in\{1, \ldots, 2 n\} .
$$

Then, the matrix $A_{\sigma}$ has the form

$$
A_{\sigma}(\tau, \lambda)=\left[\begin{array}{cccccc}
0 & 0 & 0 & 0 & 0 \ldots & 0 \\
0 & I_{d} a_{2,2} \tau_{2} & I_{d} a_{23} \tau_{2}^{1 / 2} \tau_{3}^{1 / 2} & I_{d} a_{2,4} \tau_{2}^{1 / 2} \tau_{4}^{1 / 2} & \ldots & I_{d} a_{2,2 n} \tau_{2}^{1 / 2} \tau_{2 n}^{1 / 2} \\
0 & I_{d} a_{3,2} \tau_{3}^{1 / 2} \tau_{2}^{1 / 2} & I_{d} a_{3,3} \tau_{3} & I_{d} a_{34} \tau_{3}^{1 / 2} \tau_{4}^{1 / 2} & \ldots & I_{d} a_{3,2 n} \tau_{3}^{1 / 2} \tau_{2 n}^{1 / 2} \\
\ldots & \ldots & \ldots & \ldots & \ldots & \ldots \\
0 & I_{d} a_{2 n, 2} \tau_{2 n}^{1 / 2} \tau_{2}^{1 / 2} & I_{d} a_{2 n, 3} \tau_{2 n}^{1 / 2} \tau_{3}^{1 / 2} & I_{d} a_{2 n, 4} \tau_{2 n}^{1 / 2} \tau_{4}^{1 / 2} & \ldots & I_{d} a_{2 n, 2 n} \tau_{2 n}
\end{array}\right] .
$$

Here, 0 and $I_{d}$ are the null and identity $d \times d$ matrices respectively.

We will show the following.

Proposition 3.4 We have

$$
\begin{aligned}
c_{n, \varepsilon}(t, \xi) & =\left(\frac{\varepsilon^{2}}{(2 \pi)^{d / 2}}\right)^{n} \sum_{\sigma} \int_{1}^{+\infty} \frac{s\left(\lambda_{r_{1}}\right) d \lambda_{r_{1}}}{\lambda_{r_{1}}^{\gamma+d}} \ldots \int_{1}^{+\infty} \frac{s\left(\lambda_{r_{n}}\right) d \lambda_{r_{n}}}{\lambda_{r_{n}}^{\gamma+d}} \int_{\tilde{\Delta}_{2 n}\left(t / \varepsilon^{2}\right)} d \tau_{1,2 n} \\
& \times \operatorname{det}\left(I_{2 n d}+i A_{\sigma}(\tau, \lambda)\right)^{-1 / 2} \exp \left\{-\frac{1}{2}\left(C_{\sigma}(\tau, \lambda) \Xi(\tau), \Xi(\tau)\right)_{\mathbb{R}^{2 n d}}\right\},
\end{aligned}
$$

with the matrix

$$
C_{\sigma}(\tau, \lambda)=A_{\sigma}(\tau, \lambda)-i\left(I_{2 n d}+i A_{\sigma}(\tau, \lambda)\right)^{-1} A_{\sigma}^{2}(\tau, \lambda)=\left(I_{2 n d}+i A_{\sigma}(\tau, \lambda)\right)^{-1} A_{\sigma}(\tau, \lambda)
$$

and

$$
\Xi^{T}(\tau):=\left[\tau_{1}^{1 / 2} \xi, \ldots, \tau_{2 n}^{1 / 2} \xi\right]
$$

\section{The proof of Proposition 3.4: the case $\xi=0$}

We will first consider the special case $\xi=0$. For simplicity of notation, we let $s(\lambda)=1$, as the case of a general non-negative bounded function $s(\lambda)$ is essentially identical. We have

$$
c_{n, \varepsilon}:=c_{n, \varepsilon}(t, 0)=\varepsilon^{2 n} \mathbb{M}\left\{\int_{1}^{+\infty} \frac{d \lambda}{\lambda^{\gamma}} \int_{0}^{t / \varepsilon^{2}} d s \int_{0}^{t / \varepsilon^{2}} d s^{\prime} \int_{\mathbb{R}^{d}} e^{i^{3 / 2} p \cdot\left(B_{s}-B_{s^{\prime}}\right)} e^{-\lambda^{2}|p|^{2} / 2} d p\right\}^{n} .
$$


Performing integration over $p$ in (3.44), we obtain

$$
c_{n, \varepsilon}=\varepsilon^{2 n} \mathbb{M}\left\{\frac{1}{(2 \pi)^{d / 2}} \int_{1}^{+\infty} \frac{d \lambda}{\lambda^{\gamma+d}} \int_{0}^{t / \varepsilon^{2}} d s \int_{0}^{t / \varepsilon^{2}} d s^{\prime} \exp \left\{-\frac{i}{2 \lambda^{2}}\left|B_{s}-B_{s^{\prime}}\right|^{2}\right\}\right\}^{n} .
$$

This may be re-written as

$$
\begin{aligned}
c_{n, \varepsilon} & =\left(\frac{\varepsilon^{2}}{(2 \pi)^{d / 2}}\right)^{n} \int_{1}^{+\infty} \frac{d \lambda_{1}}{\lambda_{1}^{(\gamma+d) / 2}} \ldots \int_{1}^{+\infty} \frac{d \lambda_{2 n}}{\lambda_{2 n}^{(\gamma+d) / 2}} \prod_{j=1}^{n} \delta\left(\lambda_{2 j-1}-\lambda_{2 j}\right) \\
& \times \int_{0}^{t / \varepsilon^{2}} d s_{1} \ldots \int_{0}^{t / \varepsilon^{2}} d s_{2 n} \mathbb{M} \exp \left\{-\sum_{k=1}^{n} \frac{i}{2 \lambda_{2 k}^{2}}\left|B_{s_{2 k-1}}-B_{s_{2 k}}\right|^{2}\right\} .
\end{aligned}
$$

Once again, for each $\left(s_{1}, \ldots, s_{2 n}\right)$ we re-arrange the times in the increasing order and obtain

$$
\begin{aligned}
c_{n, \varepsilon}=\left[\frac{\varepsilon^{2}}{(2 \pi)^{d / 2}}\right]^{n} & \sum_{\sigma} \int_{1}^{+\infty} \frac{d \lambda_{1}}{\lambda_{1}^{(\gamma+d) / 2}} \ldots \int_{1}^{+\infty} \frac{d \lambda_{2 n}}{\lambda_{2 n}^{(\gamma+d) / 2}} \prod_{j=1}^{n} \delta\left(\lambda_{\sigma(2 j-1)}-\lambda_{\sigma(2 j)}\right) \\
& \times \int_{\Delta_{2 n}\left(t / \varepsilon^{2}\right)} d s_{1,2 n} \mathbb{M}\left\{\exp \left\{-\sum_{k=1}^{n} \frac{i}{2 \lambda_{\sigma(2 k)}^{2}}\left|B_{s_{\sigma(2 k-1)}}-B_{s_{\sigma(2 k)}}\right|^{2}\right\}\right\} .
\end{aligned}
$$

Here, the summation extends over all possible permutations $\sigma$ of the set $\{1, \ldots, 2 n\}$ and, as we recall:

$$
\Delta_{2 n}\left(t / \varepsilon^{2}\right)=\left\{0 \leq s_{1} \leq s_{2} \leq \cdots \leq s_{2 n} \leq t / \varepsilon^{2}\right\} .
$$

In order to evaluate the expectation with respect to the (increments) of the Brownian motion in (3.46), we will use Lemma 3.2 with $f \equiv 1$ and obtain

$$
\left(\frac{1}{2 \pi}\right)^{n / 2} \int_{\mathbb{R}^{n}} \exp \left\{-\frac{1}{2}\left(|y|^{2}+z(A y, y)_{\mathbb{R}^{n}}\right)\right\} d y=\left[\operatorname{det}\left(I_{n}+z A\right)\right]^{-1 / 2} .
$$

for any $A$ an $n \times n$ symmetric, positive definite matrix and $\operatorname{Re} z \geq 0$.

We now rewrite (3.46), using the independence of the increments of the Brownian motion, in the form

$$
\begin{aligned}
c_{n, \varepsilon}=\left(\frac{\varepsilon^{2}}{(2 \pi)^{d / 2}}\right)^{n} \sum_{\sigma} \int_{1}^{+\infty} \frac{d \lambda_{1}}{\lambda_{1}^{(\gamma+d) / 2}} \cdots \int_{1}^{+\infty} \frac{d \lambda_{2 n}}{\lambda_{2 n}^{(\gamma+d) / 2}} \prod_{j=1}^{n} \delta\left(\lambda_{\ell_{j}}-\lambda_{r_{j}}\right) \int_{\tilde{\Delta}_{2 n}\left(t / \varepsilon^{2}\right)} d \tau_{1,2 n} \\
\times \int_{\mathbb{R}^{2 n d}} \exp \left\{-\sum_{k=1}^{n} \frac{i}{2 \lambda_{r_{k}}^{2}}\left|\sum_{j=\ell_{k}+1}^{r_{k}} y_{j} \tau_{j}^{1 / 2}\right|^{2}\right\}, \prod_{m=1}^{2 n}\left[\frac{1}{(2 \pi)^{d / 2}} \exp \left\{-\frac{\left|y_{m}\right|^{2}}{2}\right\}\right] d y_{1,2 n}
\end{aligned}
$$

with

$$
\tilde{\Delta}_{n}(t):=\left[\left(\tau_{1}, \ldots, \tau_{n}\right): \sum_{j=1}^{n} \tau_{j} \leq t, \tau_{j} \geq 0, j=1, \ldots, n\right]
$$


We set here $d \tau_{1,2 n}:=d \tau_{1} \ldots d \tau_{2 n}$ and $d y_{1,2 n}=d y_{1} \ldots d y_{2 n}, s_{0}:=0$. Using Lemma 3.2 , we obtain

$$
\begin{aligned}
c_{n, \varepsilon}=\left(\frac{\varepsilon^{2}}{(2 \pi)^{d / 2}}\right)^{n} & \sum_{\sigma} \int_{1}^{+\infty} \frac{d \lambda_{1}}{\lambda_{1}^{(\gamma+d) / 2}} \ldots \int_{1}^{+\infty} \frac{d \lambda_{2 n}}{\lambda_{2 n}^{(\gamma+d) / 2}} \prod_{j=1}^{n} \delta\left(\lambda_{\ell_{j}}-\lambda_{r_{j}}\right) \\
& \times \int_{\tilde{\Delta}_{2 n}\left(t / \varepsilon^{2}\right)}\left[\operatorname{det}\left(I_{2 n d}+i A_{\sigma}(\tau, \lambda)\right)\right]^{-1 / 2} d \tau_{1,2 n} .
\end{aligned}
$$

This is exactly (3.41) for $\xi=0$.

\section{The case of a general $\xi$}

We now extend representation (3.48) to non-vanishing $\xi$. The computation is slightly more tedious but quite straightforward. To abbreviate, we again write $c_{n, \varepsilon}=c_{n, \varepsilon}(t, \xi)$. We still assume for simplicity of the notation that $s(\lambda) \equiv 1$ :

$$
c_{n, \varepsilon}=\varepsilon^{2 n} \mathbb{M}\left\{\int_{1}^{+\infty} \frac{d \lambda}{\lambda^{\gamma}} \int_{0}^{t / \varepsilon^{2}} d s \int_{0}^{t / \varepsilon^{2}} d s^{\prime} \int_{\mathbb{R}^{d}} e^{i\left(B_{s}^{(\xi)}-B_{s^{\prime}}^{(\xi)}\right) \cdot p} e^{-\lambda^{2}|p|^{2} / 2} d p\right\}^{n} .
$$

Performing integration over $p$ in (3.49), we obtain

$$
\begin{aligned}
& c_{n, \varepsilon}=\left(\frac{\varepsilon^{2}}{(2 \pi)^{d / 2}}\right)^{n} \mathbb{M}\left[\int_{1}^{+\infty} \frac{d \lambda}{\lambda^{\gamma+d}} \int_{0}^{t / \varepsilon^{2}} d s \int_{0}^{t / \varepsilon^{2}} d s^{\prime} \exp \left\{-\frac{1}{2 \lambda^{2}}\left(B_{s}^{(\xi)}-B_{s^{\prime}}^{(\xi)}\right)^{2}\right\}\right]^{n} \\
& =\left(\frac{\varepsilon^{2}}{(2 \pi)^{d / 2}}\right)^{n} \int_{1}^{+\infty} \frac{d \lambda_{1}}{\lambda_{1}^{(\gamma+d) / 2}} \cdots \int_{1}^{+\infty} \frac{d \lambda_{2 n}}{\lambda_{2 n}^{(\gamma+d) / 2}} \int_{0}^{t / \varepsilon^{2}} d s_{1} \ldots \int_{0}^{t / \varepsilon^{2}} d s_{2 n} \prod_{k=1}^{n} \delta\left(\lambda_{2 k}-\lambda_{2 k-1}\right) \\
& \quad \times \exp \left\{-\frac{|\xi|^{2}}{2} \sum_{k=1}^{n}\left(\frac{s_{2 k-1}-s_{2 k}}{\lambda_{2 k}}\right)^{2}\right\} \\
& \quad \times \mathbb{M}\left\{\exp \left\{-\frac{i}{2} \sum_{k=1}^{n} \lambda_{2 k}^{-2}\left|B_{s_{2 k}}-B_{s_{2 k-1}}\right|^{2}-\sqrt{i} \xi \cdot\left[\sum_{k=1}^{n} \lambda_{2 k}^{-2}\left(s_{2 k}-s_{2 k-1}\right)\left(B_{s_{2 k}}-B_{s_{2 k-1}}\right)\right]\right\}\right\} .
\end{aligned}
$$

Re-arranging again the times $s_{j}$ in the increasing order, we obtain

$$
\begin{aligned}
& c_{n, \varepsilon}=\left(\frac{\varepsilon^{2}}{(2 \pi)^{d / 2}}\right)^{n} \sum_{\sigma} \int_{1}^{+\infty} \frac{d \lambda_{1}}{\lambda_{1}^{(\gamma+d) / 2}} \cdots \int_{1}^{+\infty} \frac{d \lambda_{2 n}}{\lambda_{2 n}^{(\gamma+d) / 2}} \prod_{k=1}^{n} \int_{\Delta_{2 n}\left(t / \varepsilon^{2}\right)} d \tau_{1,2 n} \delta\left(\lambda_{\sigma(2 k)}-\lambda_{\sigma(2 k-1)}\right) \\
& \times \exp \left\{-\frac{|\xi|^{2}}{2} \sum_{k=1}^{n}\left(\frac{s_{\sigma(2 k)}-s_{\sigma(2 k-1)}}{\lambda_{\sigma(2 k)}}\right)^{2}\right\} \mathbb{M}\left\{\operatorname { e x p } \left\{-\frac{i}{2} \sum_{k=1}^{n} \lambda_{\sigma(2 k)}^{-2} \mid B_{s_{\sigma(2 k)}}-B_{\left.s_{\sigma(2 k-1)}\right|^{2}}\right.\right. \\
& \left.\left.-\sqrt{i} \xi \cdot\left[\sum_{k=1}^{n} \lambda_{\sigma(2 k)}^{-2}\left(s_{\sigma(2 k)}-s_{\sigma(2 k-1)}\right)\left(B_{s_{\sigma(2 k)}}-B_{s_{\sigma(2 k-1)}}\right)\right]\right\}\right\} .
\end{aligned}
$$


Using the formula for the joint probability density of the random vector $\left(B_{s_{\sigma(1)}}, \ldots, B_{s_{\sigma(2 n)}}\right)$ leads to

$$
\begin{aligned}
& c_{n, \varepsilon}=\left(\frac{\varepsilon^{2}}{(2 \pi)^{d / 2}}\right)^{n} \sum_{\sigma} \int_{1}^{+\infty} \frac{d \lambda_{r_{1}}}{\lambda_{r_{1}}^{\gamma+d}} \ldots \int_{1}^{+\infty} \frac{d \lambda_{r_{n}}}{\lambda_{r_{n}}^{\gamma+d}} \int_{\Delta_{2 n}\left(t / \varepsilon^{2}\right)} d s_{1,2 n} \\
& \times \exp \left\{-\frac{|\xi|^{2}}{2} \sum_{k=1}^{n}\left(\frac{s_{\sigma(2 k)}-s_{\sigma(2 k-1)}}{\lambda_{\sigma(2 k)}}\right)^{2}\right\} \int_{\mathbb{R}^{2 d n}} \exp \left\{-\frac{i}{2} \sum_{k=1}^{n} \lambda_{r_{k}}^{-2}\left|\sum_{j=\ell_{k}+1}^{r_{k}} y_{j}\left(s_{j}-s_{j-1}\right)^{1 / 2}\right|^{2}\right. \\
& \left.-\sqrt{i} \xi \cdot\left[\sum_{k=1}^{n} \lambda_{r_{k}}^{-2}\left(s_{r_{k}}-s_{\ell_{k}}\right)\left(\sum_{j=\ell_{k}+1}^{r_{k}} y_{j}\left(s_{j}-s_{j-1}\right)^{1 / 2}\right)\right]\right\} \prod_{\ell=1}^{2 n}\left[\frac{1}{(2 \pi)^{d / 2}} \exp \left\{-\frac{\left|y_{\ell}\right|^{2}}{2}\right\}\right] d y_{1,2 n} .
\end{aligned}
$$

Changing variables $\tau_{j}:=s_{j}-s_{j-1}$ we obtain

$$
\begin{aligned}
& c_{n, \varepsilon}=\left(\frac{\varepsilon^{2}}{(2 \pi)^{d / 2}}\right)^{n} \sum_{\sigma} \int_{1}^{+\infty} \frac{d \lambda_{r_{1}}}{\lambda_{r_{1}}^{\gamma+d}} \ldots \int_{1}^{+\infty} \frac{d \lambda_{r_{n}}}{\lambda_{r_{n}}^{\gamma+d}} \int_{\tilde{\Delta}_{2 n}\left(t / \varepsilon^{2}\right)} d \tau_{1,2 n} \\
& \times \exp \left\{-\frac{1}{2}\left(A_{\sigma}(\tau, \lambda) \Xi(\tau) \cdot \Xi(\tau)\right)\right\} \int_{\mathbb{R}^{2 d n}} \exp \left\{-\frac{1}{2}\left(\left(I_{2 n d}+i A_{\sigma}(\tau, \lambda)\right) y \cdot y\right)\right\} \\
& \times \exp \left\{-\sqrt{i}\left(A_{\sigma}(\tau, \lambda) \Xi(\tau) \cdot y\right)\right\} \frac{d y_{1,2 n}}{(2 \pi)^{n d}} .
\end{aligned}
$$

Here, $A_{\sigma}(\tau, \lambda)$ are the $2 n d \times 2 n d$ block matrices as in (3.58), and $\Xi(\tau)$ is as in (3.43). Let us re-write the $y$-integral using formula (3.21):

$$
\begin{aligned}
& \int_{\mathbb{R}^{2 d n}} \exp \left\{-\frac{1}{2}\left(\left(I_{2 n d}+i A_{\sigma}(\tau, \lambda)\right) y \cdot y\right)\right\} \exp \left\{-\sqrt{i}\left(A_{\sigma}(\tau, \lambda) \Xi(\tau) \cdot y\right)\right\} \frac{d y_{1,2 n}}{(2 \pi)^{n d}} \\
& =\left[\operatorname{det}\left(I_{2 n d}+i A_{\sigma}(\tau, \lambda)\right)\right]^{-1 / 2} \int_{\mathbb{R}^{2 n d}} e^{-|x|^{2} / 2} \exp \left\{-\sqrt{i}\left(A_{\sigma}(\tau, \lambda) \Xi(\tau) \cdot\left(I_{2 n d}+i A_{\sigma}(\tau, \lambda)\right)^{-1 / 2} x\right)\right\} d x \\
& =\left[\operatorname{det}\left(I_{2 n d}+i A_{\sigma}(\tau, \lambda)\right)\right]^{-1 / 2} \\
& \times \exp \left\{\frac{i}{2}\left(\left(I_{2 n d}+i A_{\sigma}(\tau, \lambda)\right)^{-1 / 2} A_{\sigma}(\tau, \lambda) \Xi(\tau) \cdot\left(I_{2 n d}+i A_{\sigma}(\tau, \lambda)\right)^{-1 / 2} A_{\sigma}(\tau, \lambda) \Xi(\tau)\right)\right\}
\end{aligned}
$$

Using this in (3.52) gives (3.41), finishing the proof of Proposition 3.4.

\section{The proof of Proposition 3.3}

We now use representation (3.41) for $c_{n, \varepsilon}$ in order to obtain the bound (3.33) in Proposition 3.3. Once again, we first consider the simpler case $\xi=0$, and then the general case.

\section{The estimate for $\xi=0$}

The main step in the proof is the following lower bound. 
Proposition 3.5 For any permutation $\sigma$ we have

$$
\left|\operatorname{det}\left(I+i A_{\sigma}(\tau, \lambda)\right)\right| \geq \frac{1}{2^{d n / 2}} \prod_{k=1}^{n}\left(1+\frac{\tau_{r_{k}}}{\lambda_{r_{k}}^{2}}\right)^{d}
$$

Before proving this proposition, let us show how it implies the required estimate on $c_{n, \varepsilon}$ for $\xi=0$. Combining (3.41) and (3.54) implies that there exists $C>0$ such that

$$
\left|c_{n, \varepsilon}\right| \leq C^{n} \varepsilon^{2 n} \sum_{\sigma} \int_{1}^{+\infty} \frac{d \lambda_{r_{1}}}{\lambda_{r_{1}}^{\gamma+d}} \ldots \int_{1}^{+\infty} \frac{d \lambda_{r_{n}}}{\lambda_{r_{n}}^{\gamma+d}} \int_{\sum_{k=1}^{n} \tau_{\ell_{k}} \leq t / \varepsilon^{2}, \tau_{\ell_{k}} \geq 0} d \tau_{\ell_{1}} \ldots d \tau_{\ell_{n}} \prod_{k=1}^{n} \int_{0}^{+\infty} \frac{d \tau_{r_{k}}}{\left(1+\lambda_{r_{k}}^{-2} \tau_{r_{k}}\right)^{d / 2}}
$$

for all $\varepsilon>0$ and $n \geq 0$. Changing variables $\tau_{r_{k}}^{\prime}:=\lambda_{r_{k}}^{-2} \tau_{r_{k}}$ we obtain

$$
\begin{aligned}
& \left|c_{n, \varepsilon}\right| \leq C^{n} \varepsilon^{2 n} \sum_{\sigma} \int_{1}^{+\infty} \frac{d \lambda_{r_{1}}}{\lambda_{r_{1}}^{\gamma+d-2}} \ldots \int_{1}^{+\infty} \frac{d \lambda_{r_{n}}}{\lambda_{r_{n}}^{\gamma+d-2}} \int_{\sum_{k=1}^{n} \tau_{\ell_{k}} \leq t / \varepsilon^{2}, \tau_{\ell_{k}} \geq 0} d \tau_{\ell_{1}} \ldots d \tau_{\ell_{n}} \\
& \times \prod_{k=1}^{n} \int_{0}^{+\infty} \frac{d \tau_{r_{k}}}{\left(1+\tau_{r_{k}}\right)^{d / 2}} \leq(2 n) ! \frac{(C t)^{n}}{n !} \leq n !(C t)^{n},
\end{aligned}
$$

provided that $\gamma>3-d$, so that (3.33) holds. This proves Proposition 3.3 when $\xi=0$, except for the proof of Proposition 3.5.

\section{The proof of Proposition 3.5}

In order to describe the matrix $A_{\sigma}(\tau, \lambda)$ more explicitly, we make a change of variables:

$$
z_{l_{k}}=y_{l_{k}} \in \mathbb{R}^{d}, \text { and } z_{r_{k}}=\frac{1}{\lambda_{r_{k}}} \sum_{j=\ell_{k}+1}^{r_{k}} y_{j}\left(s_{j}-s_{j-1}\right)^{1 / 2} \in \mathbb{R}^{d}, \quad k=1, \ldots, n
$$

so that

$$
\left(A_{\sigma}(\tau, \lambda) y, y\right)_{\mathbb{R}^{2 d n}}=\sum_{k=1}^{n}\left|z_{r_{k}}(\tau, \lambda)\right|^{2}=\left(P_{\sigma} z, z\right)=\left(P_{\sigma} L(\tau, \lambda) y, L(\tau, \lambda) y\right) .
$$

Here $P_{\sigma}$ is the projection matrix onto the $r_{k}$-components, and $L(\tau, \lambda)$ is the matrix relating $z$ and $y$ : where $z=L(\tau, \lambda) y$. Thus, the matrix $A_{\sigma}$ has the form

$$
A_{\sigma}(\tau, \lambda)=L^{T}(\tau, \lambda) P_{\sigma} L(\tau, \lambda) .
$$

To get an expression for the change of variables matrix $L(\tau, \lambda)$, set $\rho_{\ell_{k}, j}:=\delta_{\ell_{k}, j}$, and

$$
\rho_{r_{k}, j}:=\left\{\begin{array}{l}
0, \quad \text { when } j>r_{k}, \text { or } 1 \leq j \leq \ell_{k} \\
1, \quad \text { when } \ell_{k}<j \leq r_{k},
\end{array} .\right.
$$


With this notation, the lower-triangular matrix $L(\tau, \lambda)$ has the form

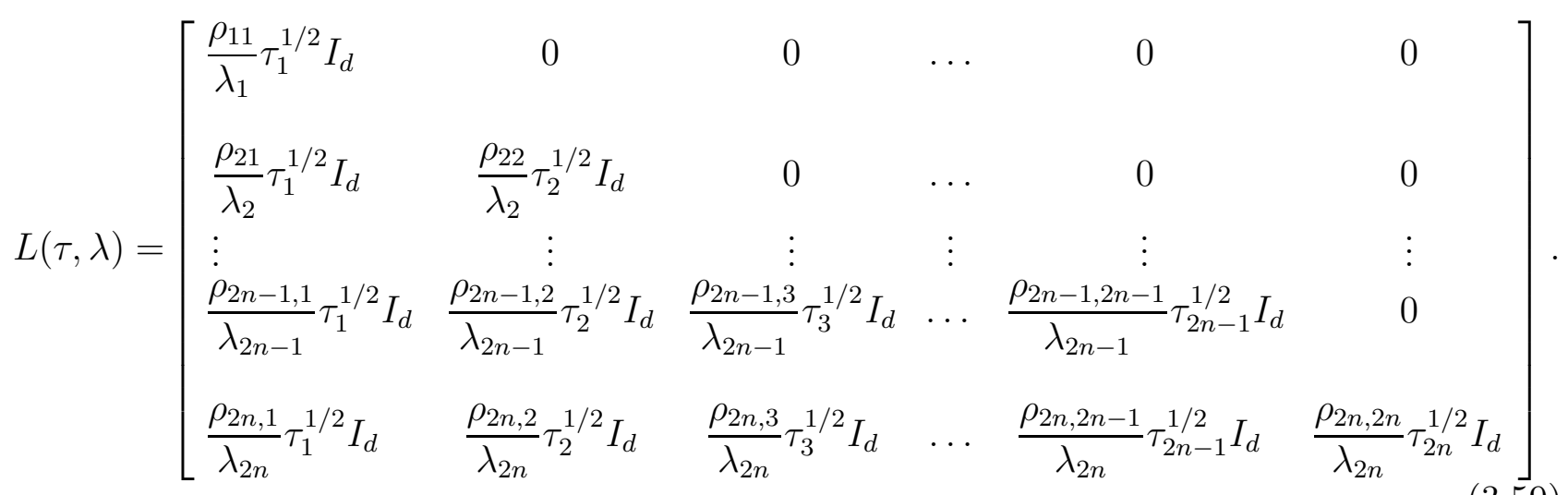

The matrices $A_{\sigma}, L$ and $P_{\sigma}$ are all block matrices, with $d \times d$ blocks, which are multiples of the identity matrix $I_{d}$. The matrix $A_{\sigma}$ is symmetric and non-negative so $\operatorname{det}\left(I_{2 n d}+i A_{\sigma}\right)$ is the product $\prod_{j=1}^{2 n d}\left(1+i \mu_{j}\right)$, where $\mu_{j}$ are the eigenvalues of $A_{\sigma}$. It is easy to see that $\mu_{j}$ are the eigenvalues of the matrix $2 n \times 2 n$ matrix $A_{\sigma}^{r}$ obtained by reducing each $d \times d$ identity block in $A_{\sigma}$ to a " $1 \times 1$ " block, except that the corresponding multiplicities are multiplied by $d$. We conclude that

$$
\operatorname{det}\left(I_{2 n d}+i A_{\sigma}\right)=\left[\operatorname{det}\left(I_{2 n}+i A_{\sigma}^{r}\right)\right]^{d} .
$$

Combining the above with (3.48), we obtain

$$
\begin{aligned}
c_{n, \varepsilon} & =\left(\frac{\varepsilon^{2}}{(2 \pi)^{d / 2}}\right)^{n} \sum_{\sigma} \int_{1}^{+\infty} \frac{d \lambda_{1}}{\lambda_{1}^{(\gamma+d) / 2}} \ldots \int_{1}^{+\infty} \frac{d \lambda_{2 n}}{\lambda_{2 n}^{(\gamma+d) / 2}} \prod_{j=1}^{n} \delta\left(\lambda_{\ell_{j}}-\lambda_{r_{j}}\right) \\
& \times \int_{\tilde{\Delta}_{2 n}\left(t / \varepsilon^{2}\right)}\left[\operatorname{det}\left(I_{2 n}+i A_{\sigma}^{r}(\tau, \lambda)\right)\right]^{-d / 2} d \tau_{1,2 n} .
\end{aligned}
$$

The reduced matrix $A_{\sigma}^{r}$ has the form as in (3.58):

$$
A_{\sigma}^{r}(\tau, \lambda)=L_{r}^{T}(\tau, \lambda) P_{r}(\sigma) L_{r}(\tau, \lambda)
$$

where $P_{r}(\sigma)$ is the projection on the (now scalar) $r_{k}$-components, and $L_{r}(\tau, \lambda)$ has the same form (3.59) as $L(\tau, \lambda)$ except that each $d \times d$ identity block is contracted to a scalar. Thus, Proposition 3.5 is a consequence of (3.60) and the following lemma.

Lemma 3.6 For any permutation $\sigma$ we have

$$
\left|\operatorname{det}\left(I_{2 n}+i A_{\sigma}^{r}(\tau, \lambda)\right)\right| \geq \frac{1}{2^{n / 2}} \prod_{k=1}^{n}\left(1+\frac{\tau_{r_{k}}}{\lambda_{r_{k}}^{2}}\right)
$$

for all $n \geq 1,\left(\tau_{1}, \ldots, \tau_{2 n}\right) \in(0,+\infty)^{2 n}$ and $\left(\lambda_{r_{1}}, \ldots, \lambda_{r_{n}}\right) \in(0,+\infty)^{n}$. 
Proof. The non-negative symmetric $2 n \times 2 n$ matrix $A_{\sigma}^{r}(\tau, \lambda)$ has eigenvalues

$$
\gamma_{1} \geq \gamma_{2} \geq \gamma_{n}>\gamma_{n+1}=\ldots=\gamma_{2 n}=0 .
$$

In order to deal with the non-degenerate part, let us denote by $N_{r}(\tau, \lambda)$ the $n \times n$ matrix obtained from $L_{r}(\tau, \lambda)$ by removing the rows and columns that correspond to the indices $\ell_{k}$, with $k=1, \ldots, n$. We will also consider the $n \times n$ matrix

$$
a_{\sigma}^{(r)}(\tau, \lambda):=N_{r}^{T}(\tau, \lambda) N_{r}(\tau, \lambda) .
$$

Let $\mu_{1} \geq \mu_{2} \geq \ldots \geq \mu_{n} \geq 0$ be the eigenvalues of $a_{\sigma}^{(r)}(s)$. We claim that

$$
\gamma_{j} \geq \mu_{j}, \quad j=1, \ldots, n .
$$

Consider the quadratic forms $Q(\cdot)$ and $P(\cdot)$ on $\mathbb{R}^{2 n}$ and $\mathbb{R}^{n}$, respectively, that correspond to the matrices $A_{\sigma}^{r}(\tau, \lambda)$ and $a_{\sigma}^{(r)}(\tau, \lambda)$. Let $H_{n}:=\operatorname{span}\left(e_{r_{j}}, j=1, \ldots, n\right) \subset \mathbb{R}^{2 n}$, and $U: \mathbb{R}^{n} \rightarrow H_{n}$ be given by

$$
U y=\sum_{j=1}^{n} y_{j} e_{r_{j}}
$$

so that $Q(U y)=P(y)$. Note that

$$
\gamma_{1}=\sup _{x \in \mathbb{R}^{2 n},\|x\|=1} Q(x) \geq \sup _{x \in H_{n},\|x\|=1} Q(x)=\sup _{y \in \mathbb{R}^{n},\|y\|=1} P(y)=\mu_{1} .
$$

Similarly, for $1<k \leq n$, let $\mathcal{H}_{k}$ the family of all subspaces of $\mathbb{R}^{2 n}$ of dimension $k, \mathcal{H}_{k}^{\prime}$ the family of all $k$-dimensional subspaces of $H_{n}$, and $\mathcal{H}_{k}^{\prime \prime}$ the family of all $k$-dimensional subspaces of $\mathbb{R}^{n}$. Then, by Fisher's principle, see part (i) of Theorem 4, p. 318 of [18], we have

$$
\gamma_{k}=\sup _{H \in \mathcal{H}_{k}} \inf _{x \in H,\|x\|=1} Q(x) \geq \sup _{H \in \mathcal{H}_{k}^{\prime}} \inf _{x \in H,\|x\|=1} Q(x)=\sup _{H \in \mathcal{H}_{n}^{\prime \prime}} \inf _{x \in H,\|x\|=1} P(x)=\mu_{k} .
$$

We see that (3.63) holds. This argument allows us to write

$$
\begin{aligned}
& \left|\operatorname{det}\left(I+i A_{\sigma}^{r}(\tau, \lambda)\right)\right|=\prod_{k=1}^{2 n}\left|\left(1+i \gamma_{k}\right)\right| \geq \prod_{k=1}^{n}\left|\left(1+i \gamma_{n+k}\right)\right| \geq \prod_{k=1}^{n}\left|\left(1+i \mu_{k}\right)\right| \geq \prod_{k=1}^{n} \frac{1+\mu_{k}}{\sqrt{2}} \\
& =\frac{1}{2^{n / 2}}\left(1+\sum_{k=1}^{n} \sum_{1 \leq i_{1}, i_{2}, \ldots, i_{k} \leq n} \mu_{i_{1}} \mu_{i_{2}} \ldots \mu_{i_{k}}\right) .
\end{aligned}
$$

In order to re-write the summation in the right side, we use an elementary linear algebra result (see p. 88 of [9]). Recall that a $k \times k$ matrix $b$ is a principal minor of rank $k \in\{1, \ldots, n\}$ of an $n \times n$ matrix $B$, if it is obtained by removing $n-k$ different rows and columns containing the diagonal elements $b_{j_{1}, j_{1}}, \ldots, b_{j_{n-k}, j_{n-k}}$ for some $j_{1}<j_{2}<\ldots<j_{n-k}$. Then, we have

$$
\sum_{b \in \mathcal{M}_{k}(B)} \operatorname{det}(b)=\sum_{1 \leq j_{1}<j_{2}<\ldots<j_{k} \leq n} \prod_{\ell=1}^{k} \eta_{j_{\ell}} .
$$


Here, $\eta_{j}, j=1, \ldots, n$ are the eigenvalues of the matrix $B$, and $\mathcal{M}_{k}(B)$ is the collection of all $k \times k$ principal minors of the matrix $B$. Thus, (3.64) can be written as

$$
\mid \operatorname{det}\left(I+i A_{\sigma}^{r}(\tau, \lambda)\right) \geq \frac{1}{2^{n / 2}}\left(1+\sum_{b \in \mathcal{M}_{k}(B)} \operatorname{det}(b)\right) .
$$

In order to estimate the right side we will use the following lemma.

Lemma 3.7 Let $b \in \mathcal{M}_{k}\left(a_{\sigma}^{(r)}(\tau, \lambda)\right)$ be the principal minor obtained from $a_{\sigma}^{(r)}(\tau, \lambda)$ by the removal of the rows and columns that correspond to the indices $1 \leq j_{1}<j_{2}<\ldots<j_{n-k} \leq n$, then

$$
\operatorname{det}(b) \geq \prod_{j \notin\left\{j_{1}, \ldots, j_{n-k}\right\}} \frac{\tau_{r_{j}}}{\lambda_{r_{j}}^{2}}
$$

Proof. Let $\tilde{l}$ be the principal minor obtained from $N_{r}(\tau, \lambda)$ by the removal of the rows and columns that correspond to the indices $1 \leq j_{1}<j_{2}<\ldots<j_{n-k} \leq n$, and $P$ be the projection matrix onto $\operatorname{span}\left\{\mathrm{e}_{r_{j}}, j \notin\left\{j_{1}, \ldots, j_{n-k}\right\}\right\}$, then

$$
\operatorname{det}(\tilde{l})=\operatorname{det}\left(P N_{r} P+I-P\right)
$$

and

$$
\operatorname{det}\left(\tilde{l}^{T}\right)=\operatorname{det}\left(P N_{r} P P N_{r}^{T} P+I-P\right)
$$

It follows that

$$
\operatorname{det}(b)=\operatorname{det}\left(P N_{r} N_{r}^{T} P+I-P\right) \geq \operatorname{det}\left(P N_{r} P P N_{r}^{T} P+I-P\right)=\operatorname{det}\left(\tilde{l}^{T}\right),
$$

as seen by the comparison of the corresponding quadratic forms. We conclude that

$$
\operatorname{det}(b) \geq \operatorname{det}\left(\tilde{l}^{t} \tilde{l}\right)=\prod_{j \notin\left\{j_{1}, \ldots, j_{n-k}\right\}} \frac{\tau_{r_{j}}}{\lambda_{r_{j}}^{2}}
$$

finishing the proof of Lemma 3.7 .

Using (3.66) in (3.64) we conclude that

$$
\left|\operatorname{det}\left(I+i A_{\sigma}^{r}(\tau, \lambda)\right)\right| \geq \frac{1}{2^{n / 2}} \prod_{k=1}^{n}\left(1+\frac{\tau_{r_{k}}}{\lambda_{r_{k}}^{2}}\right) .
$$

This finishes the proof of Lemma 3.6, and thus also that of Proposition 3.5 , 
The case $\xi \neq 0$

As for $\xi=0$, we start with (3.41) also for $\xi \neq 0$, except now we have to take into account the contribution of the matrix $C_{\sigma}(\tau, \lambda)$. The matrix $A_{\sigma}(\tau, \lambda)$ is symmetric and nonnegative, hence $C_{\sigma}(\tau, \lambda)$ is diagonalizable with respect to the orthonormal basis of eigenvectors of $A_{\sigma}(\tau, \lambda)$ :

$$
A_{\sigma}(\tau, \lambda) f_{j}=\gamma_{j} f_{j}, \quad \gamma_{j} \geq 0
$$

and

$$
C_{\sigma}(\tau, \lambda) f_{j}=\mu_{j} f_{j}, \quad \mu_{j}=\frac{\gamma_{j}}{1+i \gamma_{j}}
$$

so that

$$
\left(C_{\sigma}(\tau, \lambda) \Xi, \Xi\right)_{\mathbb{R}^{2 n d}}=\sum_{j=1}^{2 n d} \frac{\gamma_{j}}{1+i \gamma_{j}}\left(\Xi, f_{j}\right)_{\mathbb{R}^{2 n d}}^{2}
$$

thus

$$
\left|\exp \left\{-\frac{1}{2}\left(C_{\sigma}(\tau, \lambda) \Xi, \Xi\right)_{\mathbb{R}^{2 n d}}\right\}\right|=\prod_{j=1}^{2 n d} \exp \left\{-\frac{\gamma_{j}}{2\left(1+\gamma_{j}^{2}\right)}\left(\Xi, f_{j}\right)_{\mathbb{R}^{2 n d}}^{2}\right\} \leq 1 .
$$

As a consequence, we have an estimate

$$
\left|c_{n, \varepsilon}(t, \xi)\right| \leq\left(\frac{\varepsilon^{2}}{(2 \pi)^{d / 2}}\right)^{n} \sum_{\sigma} \int_{1}^{+\infty} \frac{d \lambda_{r_{1}}}{\lambda_{r_{1}}^{\gamma+d}} \ldots \int_{1}^{+\infty} \frac{d \lambda_{r_{n}}}{\lambda_{r_{n}}^{\gamma+d}} \int_{\tilde{\Delta}_{2 n}\left(t / \varepsilon^{2}\right)}\left|\operatorname{det}\left[I_{2 n d}+i A_{\sigma}(\tau, \lambda)\right]\right|^{-1 / 2} d \tau_{1,2 n}
$$

From this point on, we can repeat verbatim the estimates for $\xi=0$, and the conclusion of Proposition 3.3 can be extended to the case $\xi \neq 0$ as well.

\section{Proof of Theorem 1.1}

Proposition 3.3 allows us to pass to the limit $\varepsilon \rightarrow 0$ termwise in the expression (3.2) for $c_{n, \varepsilon}(t, \xi)$, so that

$$
\lim _{\varepsilon \rightarrow 0+} \bar{\zeta}_{\varepsilon}(t, \xi)=\hat{\psi}_{0}(\xi) \sum_{n=0}^{+\infty} \frac{(-1)^{n}}{(2 n) ! !} \lim _{\varepsilon \rightarrow 0+} c_{n, \varepsilon}(t, \xi),
$$

provided that $t \in\left[0, t_{0}\right]$, where $t_{0}$ is so small that $C t_{0}<1$, with $C$ as in (3.33). We will again assume that $s(\lambda)=1$ to simplify the notation. Let us go back to representation (3.41):

$$
\begin{aligned}
c_{n, \varepsilon}(t, \xi) & =\left(\frac{\varepsilon^{2}}{(2 \pi)^{d / 2}}\right)^{n} \sum_{\sigma} \int_{1}^{+\infty} \frac{d \lambda_{r_{1}}}{\lambda_{r_{1}}^{\gamma+d}} \ldots \int_{1}^{+\infty} \frac{d \lambda_{r_{n}}}{\lambda_{r_{n}}^{\gamma+d}} \int_{\tilde{\Delta}_{2 n}\left(t / \varepsilon^{2}\right)} d \tau_{1,2 n} \\
& \times \operatorname{det}\left(I+i A_{\sigma}(\tau, \lambda)\right)^{-1 / 2} \exp \left\{-\frac{1}{2}\left(C_{\sigma}(\tau, \lambda) \Xi(\tau), \Xi(\tau)\right)_{\mathbb{R}^{2 n d}}\right\} .
\end{aligned}
$$


Each term appearing in the sum in the right side of $(3.73)$ is of the form

$$
\frac{1}{(2 \pi)^{n d / 2}} \int_{1}^{+\infty} \cdots \int_{1}^{+\infty} \prod_{j=1}^{n} \lambda_{r_{j}}^{-\gamma-d} d \lambda_{r_{1}, r_{n}} \int_{\tilde{\Delta}_{n}(t)} \Theta_{\varepsilon}\left(\tau_{\varepsilon} ; \sigma\right) d \tau_{\ell_{1}, \ell_{n}} .
$$

Here $d \lambda_{r_{1}, r_{n}}=d \lambda_{r_{1}} \ldots d \lambda_{r_{n}}, d \tau_{\ell_{1}, \ell_{n}}=d \tau_{\ell_{1}} \ldots d \tau_{\ell_{n}}$ and the domain of integration is

$$
\tilde{\Delta}_{n}(t ; \sigma):=\left[\left(\tau_{\ell_{1}}, \ldots, \tau_{\ell_{n}}\right): \sum_{j=1}^{n} \tau_{\ell_{j}} \leq t, \tau_{\ell_{j}} \geq 0, j=1, \ldots, n\right] .
$$

The integrand in (3.74) is defined as follows: set $\tau_{\varepsilon}:=\left(\tau_{1, \varepsilon}, \ldots, \tau_{2 n, \varepsilon}\right)$, with $\tau_{\varepsilon, \ell_{j}}:=\varepsilon^{-2} \tau_{\ell_{j}}$, and $\tau_{\varepsilon, r_{j}}^{\prime}:=\tau_{r_{j}}$. Then

$$
\Theta_{\varepsilon}\left(\tau_{\varepsilon} ; \sigma\right):=\int_{\widehat{\Delta}_{n}\left((t-\tau) / \varepsilon^{2}\right)} \operatorname{det}\left(I+i A_{\sigma}\left(\tau_{\varepsilon}, \lambda\right)\right)^{-1 / 2} \exp \left\{-\frac{1}{2}\left(C_{\sigma}\left(\tau_{\varepsilon}, \lambda\right) \Xi\left(\tau_{\varepsilon}\right), \Xi\left(\tau_{\varepsilon}\right)\right)_{\mathbb{R}^{2 n d}}\right\} d \tau_{r_{1}, r_{n}},
$$

where $\tau:=\sum_{j=1}^{n} \tau_{\ell_{j}}, d \tau_{r_{1}, r_{n}}:=d \tau_{r_{1}} \ldots d \tau_{r_{n}}$ and

$$
\widehat{\Delta}_{n}(u ; \sigma):=\left[\left(\tau_{r_{1}}, \ldots, \tau_{r_{n}}\right): \sum_{j=1}^{n} \tau_{r_{j}} \leq u, \tau_{r_{j}} \geq 0, j=1, \ldots, n\right] .
$$

We will distinguish in the computation of the limit between simple and non-simple pairings note that no such distinction was made in the estimates so far.

\section{Non-simple pairings}

Recall that the pairing $\mathfrak{e}:=\{(1,2),(3,4), \ldots,(2 n-1,2 n)\}$ is called simple.

Lemma 3.8 For any $\left(\lambda_{r_{1}}, \ldots, \lambda_{r_{n}}\right) \in(1,+\infty)^{n}$ and $\left(\tau_{1}, \ldots, \tau_{2 n}\right) \in(0,+\infty)^{2 n}$, we have

$$
\lim _{\varepsilon \rightarrow 0+}\left|\operatorname{det}\left(I+i A_{\sigma}\left(\tau_{\varepsilon}, \lambda\right)\right)\right|=+\infty,
$$

provided that $\sigma$ is a permutation such that $\mathfrak{f}(\sigma) \neq \mathfrak{e}$ (see (2.22) for the definition of the map $\mathfrak{f}$ ).

Proof. Note that if $a_{\ell, \ell} \neq 0$ for some left vertex $\ell$ of $\mathfrak{f}(\sigma)$ then choosing $y=\left(y_{1}, \ldots, y_{2 n}\right)$ with $y_{j}=0$ for $j \neq \ell$ and $y_{\ell}=e$ for some $e \in \mathbb{R}^{d}$ such that $|e|=1$, we get, using (3.36) and (3.40):

$$
\lim _{\varepsilon \rightarrow 0+}\left(A_{\sigma}\left(\tau_{\varepsilon}, \lambda\right) y, y\right)_{\mathbb{R}^{2 d n}}=\lim _{\varepsilon \rightarrow 0+} a_{\ell, \ell} \frac{\tau_{\ell}}{\varepsilon^{2}}=+\infty .
$$

It follows that the largest eigenvalue of $A_{\sigma}$ satisfies $\gamma_{2 n} \rightarrow+\infty$, as $\varepsilon \rightarrow 0+$, and (3.76) follows. On the other hand, if $\sigma$ is such that

$$
a_{\ell, \ell}=0 \quad \text { for all left vertices } \ell \text { of } \mathfrak{f}(\sigma)
$$


then, according to the defintion (3.37) of $a_{\ell, \ell}$, for any left vertex $\ell$ of $\mathfrak{f}(\sigma)$ there is no bond $\left(\ell^{\prime}, r^{\prime}\right)$ such that $\ell^{\prime}<\ell<r^{\prime}$. This implies that for all bonds we have $\ell=r-1$. Indeed, otherwise we would let $\ell$ be the smallest left vertex for which $r \neq \ell+1$. Then $\ell+1$ would have to be a left vertex for which $a_{\ell+1, \ell+1} \neq 0$, giving a contradiction to (3.77). This proves that $\mathfrak{f}(\sigma)=\mathfrak{e}$.

Since, according to (3.54), there exists a constant $C>0$ such that

$$
\left|\operatorname{det}\left(I+i A_{\sigma}\left(\tau_{\varepsilon}, \lambda\right)\right)\right|^{-1 / 2} \leq C \prod_{k=1}^{n}\left(1+\frac{\tau_{r_{k}}}{\lambda_{r_{k}}^{2}}\right)^{-d / 2}
$$

for all permutations $\sigma \in \Pi(2 n),\left(\tau_{1}, \ldots, \tau_{2 n}\right)$, and $\left(\lambda_{r_{1}}, \ldots, \lambda_{r_{n}}\right)$, we conclude by the Lebesgue dominated convergence theorem that

$$
\lim _{\varepsilon \rightarrow 0+} \Theta_{\varepsilon}\left(\tau_{\varepsilon} ; \sigma\right)=0
$$

provided that $\mathfrak{f}(\sigma) \neq \mathfrak{e}$. Using the same theorem once again in (3.74), we conclude that the limit as $\varepsilon \rightarrow 0^{+}$of the terms in (3.73) corresponding to such permutations, vanishes.

\section{Simple pairings}

Observe that for any $\sigma$ such that $\mathfrak{f}(\sigma)=\mathfrak{e}$, we have $a_{m j}=0$ if $m \neq j$ and $a_{\ell, \ell}=0$ if $\ell$ is a left vertex, in other words, if $\ell$ is odd. The matrix $A_{\sigma}$ has then a particularly simple form

$$
A_{\sigma}(\tau, \lambda)=\left[\begin{array}{cccccc}
0 & 0 & 0 & \vdots & 0 & 0 \\
0 & I_{d} \lambda_{2}^{-2} \tau_{2} & 0 & \vdots & 0 & 0 \\
0 & 0 & 0 & \vdots & 0 & 0 \\
\vdots & \vdots & \vdots & \vdots & \vdots & \vdots \\
0 & 0 & 0 & \vdots & 0 & 0 \\
0 & 0 & 0 & \vdots & 0 & I_{d} \lambda_{2 n}^{-2} \tau_{2 n}
\end{array}\right]
$$

and the matrix $C_{\sigma}$ has the form

$$
C_{\sigma}(\tau, \lambda)=\left[\begin{array}{cccccc}
0 & 0 & 0 & \vdots & 0 & 0 \\
0 & I_{d}\left(\lambda_{2}^{2}+i \tau_{2}\right)^{-1} \tau_{2} & 0 & \vdots & 0 & 0 \\
0 & 0 & 0 & \vdots & 0 & 0 \\
\vdots & \vdots & \vdots & \vdots & \vdots & \vdots \\
0 & 0 & 0 & \vdots & 0 & 0 \\
0 & 0 & 0 & \vdots & 0 & I_{d}\left(\lambda_{2 n}^{2}+i \tau_{2 n}\right)^{-1} \tau_{2 n}
\end{array}\right]
$$


Thus, we obtain for such $\sigma$ :

$$
\lim _{\varepsilon \rightarrow 0+} \Theta_{\varepsilon}\left(\tau_{\varepsilon} ; \sigma\right)=\prod_{k=1}^{n} \int_{0}^{+\infty} \frac{1}{\left(1+i \lambda_{2 k}^{-2} \tau\right)^{d / 2}} \exp \left\{-\frac{|\xi|^{2} \tau^{2}}{2\left(\lambda_{2 k}^{2}+i \tau\right)}\right\} d \tau .
$$

Since, as we already know, there are precisely $(2 n) !$ ! permutations that yield $\mathfrak{f}(\sigma) \neq \mathfrak{e}$ we conclude from (3.73) $-(3.74)$ and (3.81) that

$$
\lim _{\varepsilon \rightarrow 0+} c_{n, \varepsilon}(\xi, t)=[-2 i t r(\xi)]^{n}
$$

with

$$
r(\xi)=\frac{i}{2} \frac{1}{(2 \pi)^{d / 2}} \int_{1}^{+\infty} \frac{\kappa(\lambda, \tau) d \lambda}{\lambda^{\gamma+d-2}}, \quad \kappa(\lambda, \tau)=\int_{0}^{+\infty} \frac{1}{(1+i \tau)^{d / 2}} \exp \left\{-\frac{(\lambda|\xi| \tau)^{2}}{2(1+i \tau)}\right\} d \tau
$$

This leads to the conclusion of the theorem.

\section{Proof of Theorem 1.2}

In the setting of Theorem 1.2, we conclude that there exists $t_{0}>0$ such that

$$
\bar{\zeta}_{\varepsilon}(t, \xi)=\hat{\psi}_{0}(\xi) \sum_{n=0}^{+\infty} \frac{(-1)^{n}}{(2 n) ! !} \lim _{\varepsilon \rightarrow 0+} c_{n, \varepsilon}\left(t, \varepsilon^{\beta} \xi\right)
$$

with $c_{n, \varepsilon}(t, \xi)$ given by $(3.73)$ and $t \in\left[0, t_{0}\right]$. The same computations as in the proof of Theorem 1.1 show that

$$
\lim _{\varepsilon \rightarrow 0+} c_{n, \varepsilon}\left(t, \varepsilon^{\beta} \xi\right)=[-2 i t r(0)]^{n}, \quad n \geq 0, t \geq 0 .
$$

We conclude that for any $t \in\left[0, t_{0}\right]$

$$
\lim _{\varepsilon \rightarrow 0+} \bar{\zeta}_{\varepsilon}(t, \xi)=\bar{\zeta}(t, \xi)
$$

with $\bar{\zeta}(t, \xi)$ given by (1.23), both pointwise in $\xi$ and weakly in $L^{2}\left(\mathbb{R}^{d}\right)$. In order to show that not only the limit holds for the expectation, but actually the limit is deterministic, observe that, due to (2.13), we have

$$
\begin{aligned}
& \mathbb{E}\left\|\hat{\zeta}_{\varepsilon}(t, \cdot)-\bar{\zeta}(t, \cdot)\right\|_{L^{2}\left(\mathbb{R}^{d}\right)}^{2}=\mathbb{E}\left\|\hat{\zeta}_{\varepsilon}(t, \cdot)\right\|^{2}+\|\bar{\zeta}(t, \cdot)\|^{2}-2 \operatorname{Re}\left(\bar{\zeta}_{\varepsilon}(t, \cdot), \bar{\zeta}(t, \cdot)\right)_{L^{2}\left(\mathbb{R}^{d}\right)} \\
& =2\left\|\hat{\psi}_{0}\right\|^{2}-2 \operatorname{Re}\left(\bar{\zeta}_{\varepsilon}(t, \cdot), \bar{\zeta}(t, \cdot)\right)_{L^{2}\left(\mathbb{R}^{d}\right)} \cdot
\end{aligned}
$$

Letting $\varepsilon \rightarrow 0+$ and using (3.85) we conclude that the right hand side of (3.86) tends to

$$
2\left\|\hat{\psi}_{0}\right\|^{2}-2\|\bar{\zeta}(t, \cdot)\|_{L^{2}\left(\mathbb{R}^{d}\right)}=0
$$

which ends the proof of the theorem. 


\section{The infinite effective potential regime}

In the present section we give the proof of Theorem 1.3. Adjusting for the scaling, we can write

an analogue of (3.71), though this time the simplex $\tilde{\Delta}_{2 n}\left(t / \varepsilon^{2}\right)$ is replaced by $\tilde{\Delta}_{2 n}\left(t / \varepsilon^{2 \alpha}\right)$. Using Proposition 3.5, we arrive at the estimate:

$$
\left|c_{n, \varepsilon}(t, \xi)\right| \leq C^{n} \varepsilon^{2 n} \sum_{\sigma} \int_{\tilde{\Delta}_{n}\left(t / \varepsilon^{2 \alpha}\right)} d \tau_{\ell_{1}, \ell_{n}} \prod_{k=1}^{n}\left[\int_{1}^{+\infty} \frac{d \lambda_{r_{k}}}{\lambda_{r_{k}}^{\gamma+d}} \int_{0}^{t \varepsilon^{-2 \alpha}} \frac{d \tau_{r_{k}}}{\left(1+\lambda_{r_{k}}^{-2} \tau_{r_{k}}\right)^{d / 2}}\right] .
$$

Changing variables $\tau_{r_{k}}^{\prime}:=\lambda_{r_{k}}^{-2} \tau_{r_{k}}$ we obtain

$$
\begin{aligned}
\left|c_{n, \varepsilon}(t, \xi)\right| & \leq C^{n} \varepsilon^{2 n} \sum_{\sigma} \prod_{k=1}^{n}\left[\int_{1}^{+\infty} \frac{d \lambda_{r_{k}}}{\lambda_{r_{k}}^{\gamma+d-2}} \phi\left(\frac{t}{\lambda_{r_{k}}^{2} \varepsilon^{2 \alpha}}\right)\right] \int_{\tilde{\Delta}_{n}\left(t / \varepsilon^{2 \alpha}\right)} d \tau_{\ell_{1}, \ell_{n}} \\
& \leq(2 n) ! \frac{\left(C t \varepsilon^{2-2 \alpha}\right)^{n}}{n !}\left[\int_{1}^{+\infty} \phi\left(\frac{t}{\lambda^{2} \varepsilon^{2 \alpha}}\right) \frac{d \lambda}{\lambda^{\gamma+d-2}}\right]^{n}
\end{aligned}
$$

where

$$
\phi(u):=\int_{0}^{u} \frac{d \tau}{(1+\tau)^{d / 2}}
$$

Note that

$$
\lim _{u \rightarrow+\infty} \phi(u)=\frac{2}{d-2}, \quad d \geq 3
$$

We change variables

$$
u:=\frac{t}{\lambda^{2} \varepsilon^{2 \alpha}}, \quad k=1, \ldots, n,
$$

in the integral appearing in the right side of (4.2). Taking into account (1.25), the right side of (4.2) can be then rewritten in the form

$$
(2 n) ! \frac{\left(C t^{H}\right)^{n}}{n !}\left[\int_{0}^{t / \varepsilon^{\alpha}} \phi(u) u^{(\gamma+d-5) / 2} d u\right]^{n}
$$

for

$$
H:=\frac{5-d-\gamma}{2}
$$

and an appropriate constant $C>0$. Since $(\gamma+d-5) / 2<-1$ the integral in (4.4) converges at $+\infty$, due to (4.3). As $\phi(u) \sim u$, for $u \ll 1$ we conclude that it is also convergent close to 0 , as $(\gamma+d-3) / 2>-1$. We conclude therefore that there exists $C>0$ such that

$$
\left|c_{n, \varepsilon}(t, \xi)\right| \leq(2 n) ! \frac{\left(C t^{H}\right)^{n}}{n !}\left[\int_{0}^{t / \varepsilon^{\alpha}} \phi(u) u^{(\gamma+d-5) / 2} d u\right]^{n}, \quad n \geq 0, t>0, \varepsilon>0 .
$$




\section{Computation of the limit}

Having the uniform bound (4.6), we now compute the limit. From (3.41), we obtain

$$
\begin{aligned}
c_{n, \varepsilon}(t, \xi) & =\left(\frac{\varepsilon^{2}}{(2 \pi)^{d / 2}}\right)^{n} \sum_{\sigma} \int_{1}^{+\infty} \frac{d \lambda_{r_{1}}}{\lambda_{r_{1}}^{\gamma+d}} \ldots \int_{1}^{+\infty} \frac{d \lambda_{r_{n}}}{\lambda_{r_{n}}^{\gamma+d}} \int_{\Delta_{2 n}\left(t / \varepsilon^{2 \alpha}\right)} d \tau_{1,2 n} \\
& \times \operatorname{det}\left(I+i A_{\sigma}^{r}(\tau, \lambda)\right)^{-d / 2} \exp \left\{-\frac{\varepsilon^{2 \beta}|\xi|^{2}}{2}\left(C_{\sigma}^{r}(\tau, \lambda) \top^{1 / 2} \mathbf{1} \cdot \top^{1 / 2} \mathbf{1}\right)\right\},
\end{aligned}
$$

Here, we denote $\top:=\operatorname{diag}\left[\tau_{1}, \ldots, \tau_{2 n}\right], \mathbf{1}^{T}:=\underbrace{[1, \ldots, 1]}_{2 n}$ and

$$
C_{\sigma}^{r}(\tau, \lambda):=A_{\sigma}^{r}(\tau, \lambda)\left[I_{2 n}+i A_{\sigma}^{r}(\tau, \lambda)\right]^{-1} .
$$

We change variables $\tau_{k}^{\prime}:=\varepsilon^{2 \alpha} \tau_{k} / t, k=1, \ldots, 2 n, u_{k}:=t \lambda_{r_{k}}^{-2} \varepsilon^{-2 \alpha}, k=1, \ldots, n$, and recall that

$$
H=\frac{1}{\alpha}=\frac{5-\gamma-d}{2} .
$$

We can write then

$$
\begin{aligned}
c_{n, \varepsilon}(t, \xi) & =\left(\frac{t^{H}}{2(2 \pi)^{d / 2}}\right)^{n} \sum_{\sigma} \int_{0}^{t / \varepsilon^{2 \alpha}} u_{1}^{(\gamma+d-3) / 2} d u_{1} \ldots \int_{0}^{t / \varepsilon^{2 \alpha}} u_{n}^{(\gamma+d-3) / 2} d u_{n} \int_{\tilde{\Delta}_{2 n}(1)} d \tau_{1,2 n} \\
& \times \operatorname{det}\left(I+i \tilde{A}_{\sigma}^{r}(\tau, u)\right)^{-d / 2} \exp \left\{-\frac{t \varepsilon^{2(\beta-\alpha)}|\xi|^{2}}{2}\left(\tilde{C}_{\sigma}^{r}(\tau, u) \top^{1 / 2} \mathbf{1}, \top^{1 / 2} \mathbf{1}\right)_{\mathbb{R}^{2 n d}}\right\},
\end{aligned}
$$

where $\tilde{A}_{\sigma}^{r}(\tau, u)=\left[\tilde{a}_{j, m}\right]$ is an $2 n \times 2 n$ matrix satisfying, as in (3.37)-(3.39):

$$
\tilde{a}_{1, j}=\tilde{a}_{j, 1}=0 \quad \text { for all } j \in\{1, \ldots, 2 n\},
$$

and

$$
\tilde{a}_{j, j}:=\tau_{j} \sum_{k}^{\prime} u_{k}, \text { for each } j \in\{2, \ldots, 2 n\} .
$$

The summation above extends over those $k$-s, for which $\ell_{k}<j \leq r_{k}$. Given $m<j$ we let

$$
\tilde{a}_{m, j}=\tilde{a}_{j, m}=\left(\tau_{m} \tau_{j}\right)^{1 / 2} \sum_{k}^{\prime} u_{k}
$$

and the summation extends over those $k$-s, for which $\ell_{k}<m<j \leq r_{k}$. We also let

$$
\tilde{C}_{\sigma}^{r}(\tau, u):=\tilde{A}_{\sigma}^{r}(\tau, u)\left(I_{2 n}+i \tilde{A}_{\sigma}^{r}(\tau, u)\right)^{-1} .
$$

In the limit we get

$$
\bar{c}_{n}(t, \xi):=\lim _{\varepsilon \rightarrow 0+} c_{n, \varepsilon}(t, \xi)=\left(\frac{t^{H}}{2(2 \pi)^{d / 2}}\right)^{n} \sum_{\sigma} \int_{0}^{+\infty} u_{1}^{(\gamma+d-3) / 2} d u_{1} \ldots \int_{0}^{+\infty} u_{n}^{(\gamma+d-3) / 2} d u_{n}
$$




$$
\times \int_{\tilde{\Delta}_{2 n}(1)} d \tau_{1,2 n} \operatorname{det}\left(I+i \tilde{A}_{\sigma}^{r}(\tau, u)\right)^{-d / 2}
$$

Repeating the calculations that lead to (3.48) from (3.45), this time in the reverse order, we obtain

$$
\bar{c}_{n}(t, \xi)=\mathbb{M}\left\{\frac{t^{H}}{2(2 \pi)^{d / 2}} \int_{0}^{+\infty} u^{(\gamma+d-3) / 2} d u \int_{0}^{1} d s \int_{0}^{1} d s^{\prime} \exp \left\{-\frac{i u}{2}\left|B_{s}-B_{s^{\prime}}\right|^{2}\right\}\right\}^{n}
$$

where $B_{t}$ is the $d$-dimensional standard Brownian motion and $\mathbb{M}$ is the corresponding expectation. Using the bound (4.6) we conclude that there exists $t_{0}>0$ such that it is possible to interchange the limit as $\varepsilon \rightarrow 0+$ with the summation over $n$, as in (3.72) above. Performing the summation $\sum_{n=0}^{+\infty} \bar{c}_{n}(t, \xi)$ we arrive at (1.26) .

\section{Proof of Proposition 2.2}

The conclusion of part (i) of the proposition and formula (2.12) follow, provided we can show that

$$
\sum_{n=0}^{\infty}\left[\mathbb{E}\left|\hat{\zeta}_{n}(t, \xi)\right|^{2}\right]^{1 / 2}<+\infty
$$

We have

$$
\begin{aligned}
& \mathbb{E}\left|\hat{\zeta}_{n}(t, \xi)\right|^{2}=\frac{1}{(2 \pi)^{2 n d}} \int_{\Delta_{n}(t)} d s_{1, n} \int_{\Delta_{n}(t)} d \tilde{s}_{1, n} \int_{\mathbb{R}^{2 d n}} \mathbb{E}\left[\prod_{k=1}^{n} \hat{V}\left(d p_{k}\right) \prod_{m=1}^{n} \hat{V}^{*}\left(d \tilde{p}_{m}\right)\right] \\
& \times \hat{\psi}_{0}\left(\xi-\sum_{j=1}^{n} p_{j}\right) \hat{\psi}_{0}^{*}\left(\xi-\sum_{j=1}^{n} \tilde{p}_{j}\right) e^{i G_{n}} e^{-i \tilde{G}_{n}} \leq \frac{C^{n} t^{2 n}\left\|\hat{\psi}_{0}\right\|_{\infty}^{2}}{(n !)^{2}} \int_{\mathbb{R}^{2 d n}} \mathbb{E}\left[\prod_{k=1}^{n} \hat{V}\left(d p_{k}\right) \prod_{m=1}^{n} \hat{V}^{*}\left(d \tilde{p}_{m}\right)\right] .
\end{aligned}
$$

Here $d \tilde{s}_{1, n}=d \tilde{s}_{1} \ldots d \tilde{s}_{n}$ and $\tilde{G}_{n}$ is given by (2.11), with the variables $s_{1, n}, p_{1, n}$ replaced by $\tilde{s}_{1, n}, \tilde{p}_{1, n}$. Using the rule for the expectation of a product of $2 n$ mean zero Gaussian random variables, we may estimate the above as

$$
\mathbb{E}\left|\hat{\zeta}_{n}(t, \xi)\right|^{2} \leq \frac{C^{n} t^{2 n}(2 n-1) ! !}{n !^{2}}\left(\int_{\mathbb{R}^{d}} \hat{R}(p) d p\right)^{n}\left\|\hat{\psi}_{0}\right\|_{\infty}^{2} \leq \frac{C^{n}}{n !},
$$

with a constant $C>0$ independent of $n$, and part (i) of the proposition follows. Part (ii) is a simple consequnce of the fact that we can interchange the summation and expectation in (2.9), by virtue of the estimate obtained in the proof of part (i). This, combined with the fact that the odd moments vanish, yields (2.12). 
Concerning part (iii), let us take a smooth radially symmetric non-negative function $\theta$ such that $\theta(x)=1$ for $|x| \leq 1$ and $\theta(x)=0$ for $|x|>2$. To prove (2.13), we consider the regularization of the potential: $V_{R}(x):=\theta_{R}(x) V(x)$, with $\theta_{R}(x):=\theta(x / R)$. Let $\hat{\zeta}^{(R)}(t, \xi)$ and $\hat{\zeta}_{n}^{(R)}(t, \xi)$ be the random fields given by modifications of formulas (2.9) and (2.10), with the spectral measure $\hat{V}(d p)$ replaced by $\hat{V}_{R}(p) d p$. The function

$$
\hat{\psi}^{(R)}(t, \xi):=e^{-i t|\xi|^{2} / 2} \hat{\zeta}^{(R)}(t, \xi), \quad t \geq 0
$$

is the solution of the Schrödinger equation with the potential $V_{R}(x)$. The $L^{2}$-norm conservation for the solutions of the Schrödinger equaiton with a decaying potential means that

$$
\left\|\hat{\psi}^{(R)}(t)\right\|_{L^{2}\left(\mathbb{R}^{d}\right)}=\left\|\hat{\psi}_{0}\right\|_{L^{2}\left(\mathbb{R}^{d}\right)}
$$

thus also

$$
\left\|\hat{\zeta}^{(R)}(t)\right\|_{L^{2}\left(\mathbb{R}^{d}\right)}=\left\|\hat{\psi}_{0}\right\|_{L^{2}\left(\mathbb{R}^{d}\right)} \text { for all } t, R>0 \text { a.s. }
$$

Note that

$$
\left\|\hat{\psi}_{0}\right\|_{L^{2}\left(\mathbb{R}^{d}\right)}^{2}=\mathbb{E}\left\|\hat{\zeta}^{(R)}(t)\right\|_{L^{2}\left(\mathbb{R}^{d}\right)}^{2}=\sum_{m, n \geq 0} \mathbb{E}\left[\left(\hat{\zeta}_{n}^{(R)}(t), \hat{\zeta}_{m}^{(R)}(t)\right)_{L^{2}\left(\mathbb{R}^{d}\right)}\right] .
$$

By the Cauchy-Schwarz inequality, the absolute value of the term of the series on the right hand side is bounded from above by $\left(a_{n, R} a_{m, R}\right)^{1 / 2}$, where

$$
a_{n, R}:=\mathbb{E} \int_{\mathbb{R}^{d}}\left|\hat{\zeta}_{n}^{(R)}(t, \xi)\right|^{2} d \xi
$$

Using an analogue of (5.1) for $\hat{V}_{R}(p)$ we conclude that

$$
a_{n, R} \leq \frac{C^{n} t^{2 n}(2 n-1) ! !}{n !^{2}}\left(\int_{\mathbb{R}^{d}} \hat{R}(p) d p\right)^{n}\left\|\hat{\psi}_{0}\right\|_{L^{2}\left(\mathbb{R}^{d}\right)}^{2}
$$

with the constant $C>0$ independent of $R>0$ and $n \geq 0$. It follows that that

$$
\left\|\hat{\psi}_{0}\right\|_{L^{2}\left(\mathbb{R}^{d}\right)}^{2}=\lim _{R \rightarrow+\infty} \mathbb{E}\left\|\hat{\zeta}^{(R)}(t)\right\|_{L^{2}\left(\mathbb{R}^{d}\right)}^{2}=\sum_{m, n \geq 0} \lim _{R \rightarrow+\infty} \mathbb{E}\left[\left(\hat{\zeta}_{n}^{(R)}(t), \hat{\zeta}_{m}^{(R)}(t)\right)_{L^{2}\left(\mathbb{R}^{d}\right)}\right]
$$

It is elementary calculation to verify that

$$
\lim _{R \rightarrow+\infty} \mathbb{E}\left[\left(\hat{\zeta}_{n}^{(R)}(t), \hat{\zeta}_{m}^{(R)}(t)\right)_{L^{2}\left(\mathbb{R}^{d}\right)}\right]=\mathbb{E}\left[\left(\hat{\zeta}_{n}(t), \hat{\zeta}_{m}(t)\right)_{L^{2}\left(\mathbb{R}^{d}\right)}\right]
$$

for each $n, m \geq 0$. Therefore, the right hand side of (15.3) equals $\mathbb{E}\|\hat{\zeta}(t)\|_{L^{2}\left(\mathbb{R}^{d}\right)}^{2}$, and (2.13) follows. 


\section{References}

[1] G. Bal, T. Komorowski, L. Ryzhik, Asymptotics of the solutions of a random Schrödinger equation Archiv. Rat. Mech. Appl, 200, 613-664 (2011).

[2] J. Bourgain, On random Schrödinger operators on $\mathbb{Z}^{2}$, Discrete Contin. Dynam. Systems 8 (2002), no. $1,115$.

[3] J. Bourgain, Random lattice Schrödinger operators with decaying potential: some higher dimensional phenomena, Geometric Aspects of Functional Analysis, Lecture Notes in Math., vol. 1807, Springer, Berlin, 2003, pp. 7098.

[4] M. Butz, Dynamical self-averaging for a lattice Schrödinger equation with weak random potential, http://arxiv.org/abs/1312.6979.

[5] N.H. Bingham, C.M. Goldie and J.L. Teugels, (1987) Regular Variation. Cambridge University Press.

[6] T. Chen, Localization lengths and Boltzmann limit for the Anderson model at small disorders in dimension 3. J. Stat. Phys., 120 (1-2), 279337, 2005.

[7] T. Chen, Convergence in higher mean of a random Schrödinger to a linear Boltzmann evolution, Comm. Math. Phys. 267, 2006, 355-392.

[8] L. Erdös and H.-T. Yau, Linear Boltzmann equation as the weak coupling limit of a random Schrödinger equation, Comm. Pure Appl. Math., 53, 2000, 667-735.

[9] I.M. Gelfand, Lectures on linear algebra, Interscience Publ. (1961)

[10] L. Erdös, M. Salmhofer and H.-T. Yau, Quantum diffusion for the Anderson model in the scaling limit. Ann. Henri Poincar 8 (2007), no. 4, 621685.

[11] L. Erdös, M. Salmhofer and H.-T. Yau, Quantum diffusion of the random Schrdinger evolution in the scaling limit. II. The recollision diagrams. Comm. Math. Phys. 271 (2007), no. 1, 153.

[12] J. Fröhlich, W. De Roeck and A. Pizzo, Quantum Browinian motion in a simple model system. Comm. Math. Phys. 293(2), 361398 (2010).

[13] J. Fröhlich and J. Schenker, Quantum Brownian motion induced by thermal noise in the presence of disorder. J. Math. Phys. 57 (2016), no. 2, 023305, 17 pp.

[14] I.S. Gradshteyn and I.M. Ryzhik, Table of Integrals, Series, and Products. Seventh Edition, Academic Press (2007).

[15] T. G. Ho, L. J. Landau and A. J. Wilkins, On the weak coupling limit for a Fermi gas in a random potential. Rev. Math. Phys. 5 (1993), no. 2, 209298.

[16] S. Janson, Gaussian Hilbert spaces. Cambridge University Press (1997). 
[17] Y. Kang and J. Schenker, Diffusion of wave packets in a Markov random potential. J. Stat. Phys. 134 (2009), no. 5-6, 10051022.

[18] P. Lax, Functional Analysis, Wiley and Sons, (2002).

[19] J. Lukkarinen and H. Spohn, Kinetic limit for wave propagation in a random medium. Arch. Ration. Mech. Anal. 183 (2007), no. 1, 93162.

[20] F. Poupaud and A. Vasseur, Classical and quantum transport in random media. J. Math. Pures Appl. (9) 82 (2003), no. 6, 711748.

[21] P.D. Powell, Calculating Determinants of Block Matrices, https://arxiv.org/pdf/1112.4379.pdf

[22] I.J. Schoenberg, Metric spaces and completely monotone functions. Ann. of Math. (2) 39 (1938), no. $4,811-841$.

[23] H. Spohn, Derivation of the transport equation for electrons moving through random impurities, J. Stat. Phys., 17, 1977, 385-412.

[24] N. Zhang and G. Bal, Convergence to spde of the Schrödinger equation with large, random potential, Comm. Math. Sci., 12, 2014.

[25] N. Zhang and G. Bal, Homogenization of the Schrödinger equation with large, random potential, Stoch. Dyn., 14, 2014. 